\title{
Foci in ray pencils of general divergency
}

\section{WF Harris* and RD van Gool ${ }^{+}$}

\author{
Department of Optometry, University of Johannesburg, PO Box 524, Auckland Park, 2006 South \\ Africa
}

<wharris@uj.ac.za> and <rdvg@mweb.co.za>

\begin{abstract}
In generalized optical systems, that is, in systems which may contain thin refracting elements of asymmetric dioptric power, pencils of rays may exhibit phenomena that cannot occur in conventional optical systems. In conventional optical systems astigmatic pencils have two principal meridians that are necessarily orthogonal; in generalized systems the principal meridians can be at any angle. In fact in generalized systems a pencil may have only one principal meridian or even none at all. In contrast to the line foci in the conventional interval of Sturm line foci in generalized systems may be at any angle and there may be only one line focus or
\end{abstract}

no line foci. A conventional cylindrical pencil has a single line focus at a finite distance but it can be regarded as having a second line focus at infinity. Only in generalized systems is a single line focus possible without a second at infinity or anywhere else. The purpose of this paper is to illustrate the types of pencils possible in generalized systems. Particular attention is paid to the effect of including an antisymmetric component in the divergency of the pencil.

Key words: divergency, asymmetric divergency, non-orthogonal line foci, single line foci, generalized linear optics

\section{Introduction}

A recent paper ${ }^{1}$ describes thin lenses with asymmetric dioptric power. The lenses are built up of small prisms and the power is of a type that has no equivalent in the traditional spherocylindrical representation employed in optometry. We shall refer to optical systems which may contain such lenses as generalized optical systems. Conventional optical systems do not contain such lenses; they constitute a subset of generalized optical systems. A paper ${ }^{2}$ accompanying this one examines the nature of pencils of rays of light through generalized optical systems. The state of a pencil is represented by a $2 \times 2$ matrix, called the divergency, which may be symmetric or asymmetric. In conventional systems it is the negative of the ver- gence matrix ${ }^{3-6}$. Equations are derived ${ }^{2}$ which allow step-along divergency calculations through generalized optical systems similar to step-along vergence calculations $^{7}$ in conventional systems.

Generalized systems exhibit features which are not possible in conventional systems. A pencil of rays can have two principal meridians that are not orthogonal, or there may be only one principal meridian or even none. As a consequence line foci need not be orthogonal or there may be only one or none. ${ }^{2}$ The case with the single line focus is not equivalent to a cylindrical pencil in conventional systems; the latter has two line foci, one at a finite distance and the other at infinity. The purpose of this paper is to make use of the mathematical analysis of the accompanying paper $^{2}$ to illustrate and examine these features. 
We begin by summarizing those results in the accompanying paper ${ }^{2}$ that are needed in this paper. The simplest pencil, a pencil of parallel rays, is examined first. It provides a reference or framework for the illustrations that follow. The emphasis here will be on the effect of including an antisymmetric component in the divergency. Working in five sets of three we begin each set with a pencil which is possible in a conventional optical system and then add an antisymmetric component and, finally, double the amount of antisymmetric component. Lastly we shall examine the effect of adding an antisymmetric component to a singular (cylindrical) divergency.

Throughout this paper we shall be working in terms of divergency. Reversing the sign allows one to think in terms of a generalized vergence (Equation 39 of the accompanying paper ${ }^{2}$ ) instead.

In generalized systems principal divergencies are complex numbers. Corresponding to them are what we refer to as complex principal meridional planes, complex focal planes and complex foci. This paper restricts its attention to optical features represented by real numbers. Conventional systems have principal divergences and powers that are complex but their principal divergencies and vergences are necessarily real. ${ }^{8,9}$

As we have pointed out before ${ }^{1,2}$ one expects that the very simple model used in this context may be very limited in conventional applications. Nevertheless there are less-conventional applications we believe, an example being the nodes in nodal rays examined elsewhere ${ }^{10}$.

\section{Divergency in generalized optical systems}

For convenient reference below we summarize here results of the accompanying paper ${ }^{2}$. We use the following convention: Equation $45^{2}$, for example, will refer to Equation 45 in the accompanying paper and similarly for other numbered items.

A pencil of rays is defined in terms of its divergency $\mathbf{M}$ (Definitions $1^{2}$ and $2^{2}$ ) at a particular transverse plane. Here we shall consider only centred pencils. At a particular transverse plane the reduced inclinations $\alpha(2 \times 1)$ of the rays in a centred pencil are a linear function of their transverse positions $\mathbf{y}(2 \times 1)$. More particularly $\alpha=\mathbf{M y}$. The divergency is a $2 \times 2$ matrix which we shall often expand as (Equation $44^{2}$ )
$\mathbf{M}=M_{\mathrm{I}} \mathbf{I}+M_{\mathrm{J}} \mathbf{J}+M_{\mathrm{K}} \mathbf{K}+M_{\mathrm{L}} \mathbf{L}$

$\mathbf{I}$ is the identity matrix and $\mathbf{J}, \mathbf{K}$ and $\mathbf{L}$ are defined by Equations $45^{2}$ to $47^{2}$. Together $\mathbf{I}, \mathbf{J}, \mathbf{K}$ and $\mathbf{L}$ constitute an orthonormal basis for divergency. We shall refer to $M_{\mathrm{I}} \mathbf{I}$ as the scalar component, $M_{\mathrm{J}} \mathbf{J}$ the ortho-component, $M_{\mathrm{K}} \mathbf{K}$ the oblique component and $M_{\mathrm{L}} \mathbf{L}$ the antisymmetric component of the divergency M. $M_{\mathrm{I}}$ is the scalar coefficient of $\mathbf{M}$ and similarly for the other coefficients. $M_{\mathrm{I}} \mathbf{I}+M_{\mathrm{J}} \mathbf{J}+M_{\mathrm{K}} \mathbf{K}$ is the symmetric component of the divergency. It will be convenient to refer to $M_{\mathrm{J}} \mathbf{J}+M_{\mathrm{K}} \mathbf{K}+M_{\mathrm{L}} \mathbf{L}$ as the antiscalar component.

If the divergency of a pencil at transverse plane $\mathrm{T}$ in a homogeneous medium is $\mathbf{M}$ then its divergency at a transverse plane $\mathrm{T}^{\prime}$ a reduced distance $\zeta$ downstream is given by any of Equations $33^{2}, 36^{2}$ to $38^{2}$ provided the inverses involved in those equations exist. In those equations $\mathbf{M}_{1}$ becomes $\mathbf{M}$ and $\mathbf{M}_{2}$ becomes $\mathbf{M}^{\prime}$. In particular Equation $36^{2}$ becomes

$\mathbf{M}(\mathbf{I}+\zeta \mathbf{M})^{-1}=\mathbf{M}^{\prime}$.

We shall also make use of Equation $38^{2}$ which becomes

$\left(\mathbf{M}^{-1}+\zeta \mathbf{I}\right)^{-1}=\mathbf{M}^{\prime}$

The discriminant of the divergency is defined by (Equation 60²)

$\operatorname{dis} \mathbf{M}=4\left(M_{\mathrm{J}}^{2}+M_{\mathrm{K}}^{2}-M_{\mathrm{L}}^{2}\right)$.

According to Equation $59^{2}$ there are three cases of interest: the discriminant is positive, zero or negative. We summarize each in turn.

If $\operatorname{dis} \mathbf{M}>0 \mathrm{D}^{2}$ the pencil has two distinct principal divergencies given by (Equation $59^{2}$ )

$$
M=M_{\mathrm{I}} \pm \frac{1}{2} \sqrt{\operatorname{dis} \mathbf{M}} \text {. }
$$

Corresponding to each principal divergency there is a principal plane that lies at angle A given by (Equations $61^{2}$ and $62^{2}$ )

$$
A=\tan ^{-1} \frac{M-M_{\mathrm{I}}-M_{\mathrm{J}}}{M_{\mathrm{K}}+M_{\mathrm{L}}}
$$


if $M_{\mathrm{K}}+M_{\mathrm{L}} \neq 0 \mathrm{D}$,

$$
A=\tan ^{-1} \frac{M_{\mathrm{K}}-M_{\mathrm{L}}}{M-M_{\mathrm{I}}+M_{\mathrm{J}}}
$$

if $M \neq M_{\mathrm{I}}-M_{\mathrm{J}}$ and by (Equation $58^{2}$ )

$$
A=\frac{\pi}{2}
$$

otherwise. Where desirable we can distinguish the principal divergencies as $M_{+}$and $M_{-}$corresponding to the + and - signs in Equation 5. Similarly the corresponding angles can be distinguished as $A_{+}$and $A_{-}$. (We note that the principal meridional planes may or may not be orthogonal.) The reduced distance to corresponding focal planes can be represented by $\zeta_{+}$and $\zeta_{-}$and the orientation of corresponding focal lines by $\phi_{+}$and $\phi_{-}$. When discussing one of the principal divergencies of a pair we shall sometimes refer to the other one as the complementary principal divergency. We may do the same for other features. Thus if we are talking about the focal line at reduced distance $\zeta_{-}$, for example, then the complementary focal line is the focal line at reduced distance $\zeta_{+}$.

We consider now the case in which $\operatorname{dis} \mathbf{M}=0 \mathrm{D}^{2}$. From Equation 5 we see that there is a unique principal divergency $M=M_{\mathrm{I}}$. If $\mathbf{M}$ is also symmetric, that is, if the antisymmetric coefficient $M_{\mathrm{L}}$ is zero, then, by Equation $4, M_{\mathrm{J}}=M_{\mathrm{K}}=0 \mathrm{D}$ and $\mathbf{M}=M_{\mathrm{I}} \mathbf{I}$; every meridional plane is a principal meridional plane. On the other hand if $\mathbf{M}$ is asymmetric $M_{\mathrm{L}}$ is not zero and we see from Equation 4 that

$$
M_{\mathrm{J}}^{2}+M_{\mathrm{K}}^{2}=M_{\mathrm{L}}^{2} \text {. }
$$

Corresponding to the unique principal divergency is a unique principal meridional plane lying at an angle given by Equations 6, 7 or 8 .

Finally, when $\operatorname{dis} \mathbf{M}<0 \mathrm{D}^{2}$, there is no principal divergency or principal meridional plane.

Corresponding to a principal divergency $M$ is a focal plane at reduced distance (Equation $72^{2}$ )

$$
\zeta=-\frac{1}{M}
$$

downstream from transverse plane T. If $\zeta<0 \mathrm{~m}$ the focal plane is upstream from T. For $\operatorname{dis} \mathbf{M}>0$ $\mathrm{D}^{2}$ there is a focal line in each of the two principal meridional planes at reduced distances $\zeta_{ \pm}$. The focal line is in the complementary principal meridional plane, that is, it lies at angle (Equation $86^{2}$ )

$\phi_{ \pm}=A_{\mp}$.

For disM $=0 \mathrm{D}^{2}$ there is a unique focal plane located according to Equation 10; the focal plane contains a focal point if $M_{\mathrm{L}}=0 \mathrm{D}$ and a focal line if $M_{\mathrm{L}} \neq 0$ D. The focal line lies in the unique principal meridional plane, at angle

$\phi=A$.

\section{Pencils of null divergency}

The simplest of all pencils is shown in Figure 1. It has the form of a box with square cross-section. $\mathrm{Z}$ is a longitudinal axis. $\mathrm{T}$ is a transverse plane. $\mathrm{T}^{\prime}$ is a second transverse plane at reduced distance $\zeta$ downstream from $T$. A small solid circle marks the intersection of $\mathrm{Z}$ with $\mathrm{T}$ and an a small empty circle the intersection of $\mathrm{Z}$ and $\mathrm{T}^{\prime}$. Our primary interest is in the geometry of the rays in the system between these two transverse planes. We may refer to $T$ as the entrance plane of the system and $\mathrm{T}^{\prime}$ as the exit plane. If we imagine standing upstream from $\mathrm{T}$ and looking along $\mathrm{Z}$ we see a blue line defining the top (blue for sky) of the box, a green line defining the bottom (green for grass), a red line defining the right ( $r$ for right) and a cyan line defining the left of the box. We can think of the four coloured lines as representing a square aperture that defines which rays are transmitted between $\mathrm{T}$ and $\mathrm{T}^{\prime}$. The same colours are used to define the boundary of the pencil in transverse plane $\mathrm{T}^{\prime}$. Twenty-five rays are shown traversing the box from $\mathrm{T}$ to $\mathrm{T}^{\prime}$; they are equally spaced on a square lattice with five rows and five columns. The light is travelling from left to right and into the plane of the paper. The corner rays are shown thicker than the other rays.

At entrance plane $\mathrm{T}$ in Figure 1 the divergency of the pencil is null, that is, $\mathbf{M}=\mathbf{O} \mathrm{D}$. In keeping with Equation 2 it is also null at exit plane $\mathrm{T}^{\prime}$, and, indeed, at every transverse plane. All of the coefficients in Equation 1 are zero and the discriminant (Equation 4 ) is zero. It follows that every meridional plane is a 
principal meridional plane. We can think of there being a unique focal plane at infinity and that the focal plane contains a focal point.

For all the figures that follow in this paper Figure 1 provides a framework. The positions of the rays in entrance plane $\mathrm{T}$ of all the figures is the same as in Figure 1. For purposes of clarity and convenience the reduced distance between the two transverse planes $\mathrm{T}$ and $\mathrm{T}^{\prime}$ has been chosen as $\zeta=2 \mathrm{~m}$ for all the figures. This allows important features of the pencil to be centred at or near a reduced distance of $1 \mathrm{~m}$ in many of the examples. The square defining the boundary of the pencil in $\mathrm{T}^{\prime}$ is shown by means of a fine dashed line in (b) of all subsequent figures. The coloured lines in $\mathrm{T}^{\prime}$ in Figure 1 define a square of the same size and orientation as in the entrance plane $\mathrm{T}$. In the figures that follow we shall see that the square in $T^{\prime}$ may be rotated, magnified or otherwise altered. Data for all the figures are summarized in Table 1.

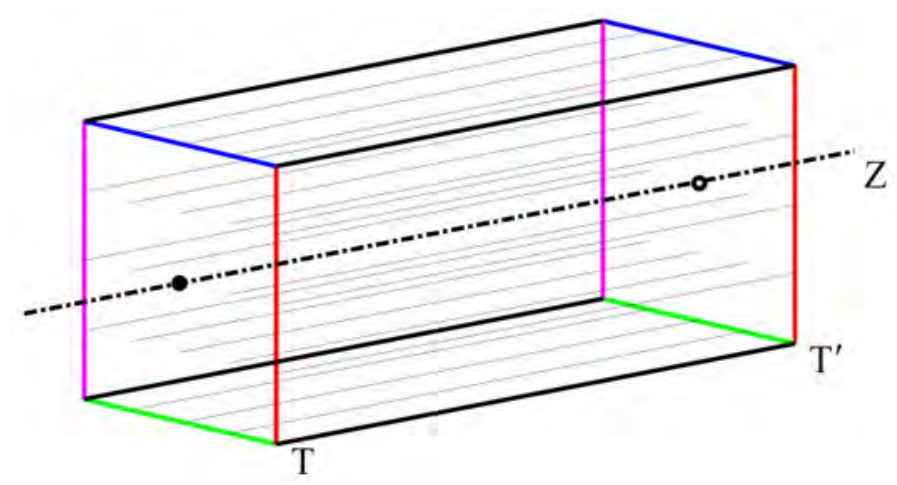

Figure 1 Pencil of null divergency at entrance plane T, that is, $\mathbf{M}=\mathbf{O}$ D. The divergency is also null at the exit plane $\mathrm{T}^{\prime}$ and everywhere else. $\mathrm{Z}$ is the longitudinal axis. Twenty-five rays are shown entering at $\mathrm{T}$ in a 5-by-5 square array. Azimuth $-43^{\circ}$ elevation $12^{\circ}$.

\section{Antisymmetric divergency}

Figures 2 and 3 show the effect of adding an increasing amount of antisymmetric component $M_{\mathrm{L}} \mathbf{L}$ to the null divergency at entrance plane $\mathrm{T}$ in Figure 1 . In Figure $2 \mathbf{L} / 4$ D is added and in Figure 3 and $\mathbf{L} / 2$ $\mathrm{D}$ is added. Thus in Figure 2 the divergency at $\mathrm{T}$ is

$$
\mathbf{M}=\frac{1}{4} \mathbf{L}=\left(\begin{array}{cc}
0 & 1 / 4 \\
-1 / 4 & 0
\end{array}\right) \mathrm{D}
$$

Figure 2(a) is the view looking along longitudinal axis $\mathrm{Z}$ from upstream of T. Right-handed torsion in the pencil is evident in (b). At $\mathrm{T}^{\prime}, 2 \mathrm{~m}$ downstream from $\mathrm{T}$, the boundary of the pencil has turned clockwise through about $26.6^{\circ}$ and expanded by the factor of about 1.12. (These numbers were calculated by means of Equation $73^{2}$.) By Equations 2 or 3 the divergency at $\mathrm{T}^{\prime}$ is

$\mathbf{M}^{\prime}=\frac{1}{10} \mathbf{I}+\frac{1}{5} \mathbf{L}=\left(\begin{array}{cc}1 / 10 & 1 / 5 \\ -1 / 5 & 1 / 10\end{array}\right)$ D.

Relative to the divergency at $\mathrm{T}$ the antisymmetric component has decreased; and the pencil has acquired a positive scalar component which is reflected in the expansion of the pencil at $\mathrm{T}^{\prime}$.

By Equation 4 the discriminant of the divergency $\mathbf{M}$ at $\mathrm{T}$ is $-1 / 4 \mathrm{D}^{2}$. Because the divergency is negative the pencil has no principal divergencies or principal meridional planes and there are no foci anywhere (hence the dashes for this case in the fourth and fifth columns of Table 1).

Adding an additional $\mathbf{L} / 4 \mathrm{D}$ to the divergency at the transverse plane $\mathrm{T}$ results in a divergency at $\mathrm{T}$ of $\mathbf{M}=\frac{1}{2} \mathbf{L}=\left(\begin{array}{cc}0 & 1 / 2 \\ -1 / 2 & 0\end{array}\right)$ D.

The pencil is shown in Figure 3. The right-handed torsion has increased relative to that in Figure 2. At $\mathrm{T}^{\prime}$ the boundary of the pencil has rotated $45^{\circ}$ clockwise and magnified by the factor $\sqrt{2}$ and, by Equations 2 or 3, its divergency is

$$
\mathbf{M}^{\prime}=\frac{1}{4} \mathbf{I}+\frac{1}{4} \mathbf{L}=\left(\begin{array}{cc}
1 / 4 & 1 / 4 \\
-1 / 4 & 1 / 4
\end{array}\right) \mathrm{D} \text {. }
$$

As in Figure 2 the discriminant of $\mathbf{M}$ is negative and, hence, there are no principal divergencies, principal meridional planes or foci.

In both Figures 2 and 3 the antisymmetric coefficient $M_{\mathrm{L}}$ of the divergency is positive and the pencil exhibits right-handed torsion. If $M_{\mathrm{L}}$ is negative the pencil has left-handed torsion equivalent to the images of Figure 2 or 3 reflected in a flat mirror. If $M_{\mathrm{L}}$ is zero then the pencil is as in Figure 1. Thus for $M_{\mathrm{L}}=0 \mathrm{D}$ the pencil is achiral (not different from its mirror image); for $M_{\mathrm{L}} \neq 0 \mathrm{D}$ the pencil is chiral (dif- 
ferent from its mirror image) and changing the sign of $M_{\mathrm{L}}$ reverses the chirality. If one ignores the square arrangement of rays the pencil is invariant under rotation about longitudinal axis $\mathrm{Z}$ in Figures 1 to 3 .

Table 1 The divergency $\mathbf{M}$ at entrance plane T, its discriminant (Equation 4), its real principal divergencies $M$ (Equation 5) and principal meridional planes $A$ (Equations 6,7 or 8), reduced distances to the focal planes $\zeta$ (Equation 10) and the divergency $\mathbf{M}^{\prime}$ (Equation 2) at exit plane $\mathrm{T}^{\prime}$. Figures 1 and 7 have point foci; in other cases where there is only one focal plane there is a single line focus in the principal meridional plane ( $\phi=A$, Equation 12); where there are two focal planes there is a focal line in each in the complementary principal meridional plane $\left(\phi_{ \pm}=A_{\mp}\right.$, Equation 11$)$.

\begin{tabular}{|c|c|c|c|c|c|}
\hline Figure & $\begin{array}{c}\text { Divergency at } \mathrm{T} \\
\text { M/D }\end{array}$ & $\begin{array}{c}\text { Discriminant } \\
\text { DisM } / \mathrm{D}^{2}\end{array}$ & $\begin{array}{c}\text { Principal meridional } \\
\text { divergency } \\
M_{+}\left\{A_{+}\right\} M_{-}\left\{A_{-}\right\}\end{array}$ & $\begin{array}{l}\text { Location of } \\
\text { focal plane } \\
\zeta_{+} / \mathrm{m} \quad \zeta_{-} / \mathrm{m}\end{array}$ & $\begin{array}{c}\text { Divergency at } \mathrm{T}^{\prime} \\
\mathbf{M}^{\prime} / \mathrm{D}\end{array}$ \\
\hline 1 & $\mathbf{O}$ & 0 & $0\{$ all $\}$ & $\infty$ & $\mathbf{O}$ \\
\hline 2 & $\frac{1}{4} \mathbf{L}$ & $-\frac{1}{4}$ & $=$ & - & $\frac{1}{10} \mathbf{I}+\frac{1}{5} \mathbf{L}$ \\
\hline 3 & $\frac{1}{2} \mathbf{L}$ & -1 & - & - & $\frac{1}{4} \mathbf{I}+\frac{1}{4} \mathbf{L}$ \\
\hline 4 & $\mathbf{J}$ & 4 & $1\{180\}-1\{90\}$ & $-1 \quad 1$ & $\frac{2}{3} \mathbf{I}-\frac{1}{3} \mathbf{J}$ \\
\hline 5 & $\mathbf{J}+\frac{1}{4} \mathbf{L}$ & $\frac{15}{4}$ & $0.968\{172.8\}-0.968\{97.2\}$ & $-1.033 \quad 1.033$ & $\frac{15}{22} \mathbf{I}-\frac{4}{11} \mathbf{J}-\frac{1}{11} \mathbf{L}$ \\
\hline 6 & $\mathbf{J}+\frac{1}{2} \mathbf{L}$ & 3 & $0.866\{165\}-0.866\{105\}$ & $-1.155 \quad 1.155$ & $\frac{3}{4} \mathbf{I}-\frac{1}{2} \mathbf{J}-\frac{1}{4} \mathbf{L}$ \\
\hline 7 & $-\mathbf{I}$ & 0 & $-1\{$ all $\}$ & 1 & I \\
\hline 8 & $-\mathbf{I}+\frac{1}{4} \mathbf{L}$ & $-\frac{1}{4}$ & - & - & $\frac{9}{10} \mathbf{I}+\frac{\mathbf{I}}{5} \mathbf{L}$ \\
\hline 9 & $-\mathbf{I}+\frac{1}{2} \mathbf{L}$ & -1 & - & - & $\frac{3}{4} \mathbf{I}+\frac{1}{4} \mathbf{L}$ \\
\hline 10 & $-\mathbf{I}+\frac{1}{4} \mathbf{J}$ & $\frac{1}{4}$ & $-0.75\{180\}-1.25\{90\}$ & $0.8 \quad 1.333$ & $\frac{7}{6} \mathbf{I}+\frac{1}{3} \mathbf{J}$ \\
\hline 11 & $-\mathbf{I}+\frac{1}{4} \mathbf{J}+\frac{1}{4} \mathbf{L}$ & 0 & $-1\{135\}$ & 1 & $\mathbf{I}+\frac{1}{4} \mathbf{J}+\frac{1}{4} \mathbf{L}$ \\
\hline 12 & $-\mathbf{I}+\frac{1}{4} \mathbf{J}+\frac{1}{2} \mathbf{L}$ & $-\frac{3}{4}$ & - & - & $\frac{11}{14} \mathbf{I}+\frac{1}{7} \mathbf{J}+\frac{2}{7} \mathbf{L}$ \\
\hline $13^{*}$ & $-\mathbf{I}+\frac{1}{2} \mathbf{J}$ & 1 & $-1.5\{90\}-0.5\{180\}$ & 0.6672 & $\infty \mathbf{I}+\infty \mathbf{J}$ \\
\hline 14 & $-\mathbf{I}+\frac{1}{2} \mathbf{J}+\frac{1}{4} \mathbf{L}$ & $\frac{3}{4}$ & $-0.567\{165\}-1.433\{105\}$ & 1.7640 .698 & $\frac{5}{2} \mathbf{I}+2 \mathbf{J}+\mathbf{L}$ \\
\hline 15 & $-\mathbf{I}+\frac{1}{2} \mathbf{J}+\frac{1}{2} \mathbf{L}$ & 0 & $-1\{135\}$ & 1 & $\mathbf{I}+\frac{1}{2} \mathbf{J}+\frac{1}{2} \mathbf{L}$ \\
\hline 16 & $-\mathbf{I}+\mathbf{J}$ & 4 & $0\{180\}-2\{90\}$ & $\infty \quad 0.5$ & $\frac{1}{3} \mathbf{I}-\frac{1}{3} \mathbf{J}$ \\
\hline 17 & $-\mathbf{I}+\mathbf{J}+\frac{1}{4} \mathbf{L}$ & $\frac{15}{4}$ & $-0.032\{172.8\}-1.968\{97.2\}$ & $31.492 \quad 0.508$ & $\frac{7}{22} \mathbf{I}-\frac{4}{11} \mathbf{J}-\frac{1}{11} \mathbf{L}$ \\
\hline 18 & $-\mathbf{I}+\mathbf{J}+\frac{1}{2} \mathbf{L}$ & 3 & $-0.134\{165\}-1.866\{105\}$ & $7.464 \quad 0.536$ & $\frac{1}{4} \mathbf{I}-\frac{1}{2} \mathbf{J}-\frac{1}{4} \mathbf{L}$ \\
\hline 19 & $-\mathbf{I}+\mathbf{J}+\frac{3}{4} \mathbf{L}$ & $\frac{7}{4}$ & $-0.339\{155.7\}-1.661\{114.3\}$ & $2.954 \quad 0.602$ & $-\frac{1}{6} \mathbf{I}-\frac{4}{3} \mathbf{J}-\mathbf{L}$ \\
\hline 20 & $-\mathbf{I}+\mathbf{J}+\mathbf{L}$ & 0 & $-1\{135\}$ & 1 & $\mathbf{I}+\mathbf{J}+\mathbf{L}$ \\
\hline 21 & $-\mathbf{I}+\mathbf{J}+\frac{5}{4} \mathbf{L}$ & $-\frac{9}{4}$ & - & - & $\frac{17}{26} \mathbf{I}+\frac{4}{13} \mathbf{J}+\frac{5}{13} \mathbf{L}$ \\
\hline
\end{tabular}

* The divergency at $\mathrm{T}^{\prime}$ in this case is better given by Equation 13. 


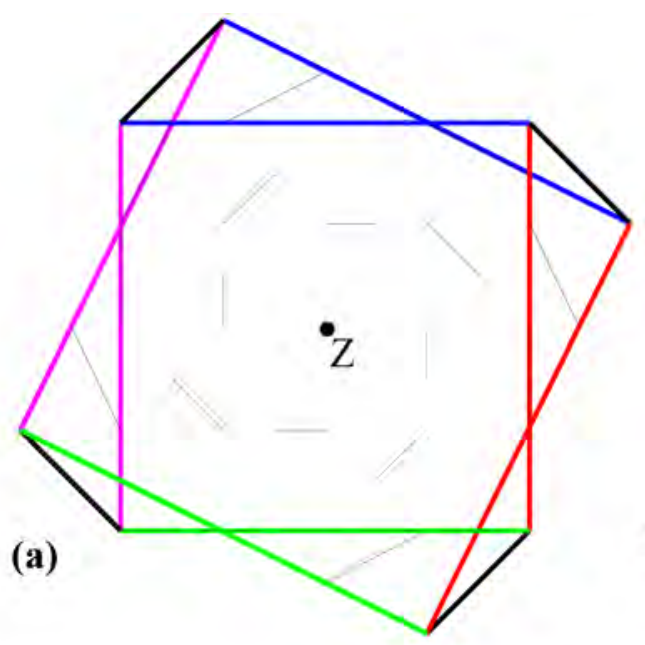

(b)

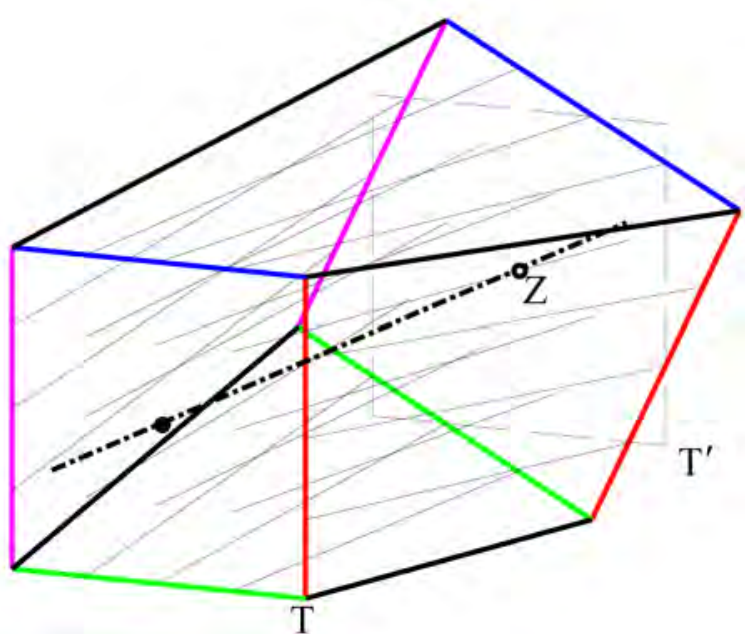

Figure 2 A pencil of divergency $\mathbf{M}=\mathbf{L} / 4 \mathrm{D}$ at entrance plane T. There are no principal meridional planes and no foci. (a) shows the view along longitudinal axis $\mathrm{Z}$ from a point upstream of the entrance plane. (b) azimuth $-64^{\circ}$, elevation $12^{\circ}$.

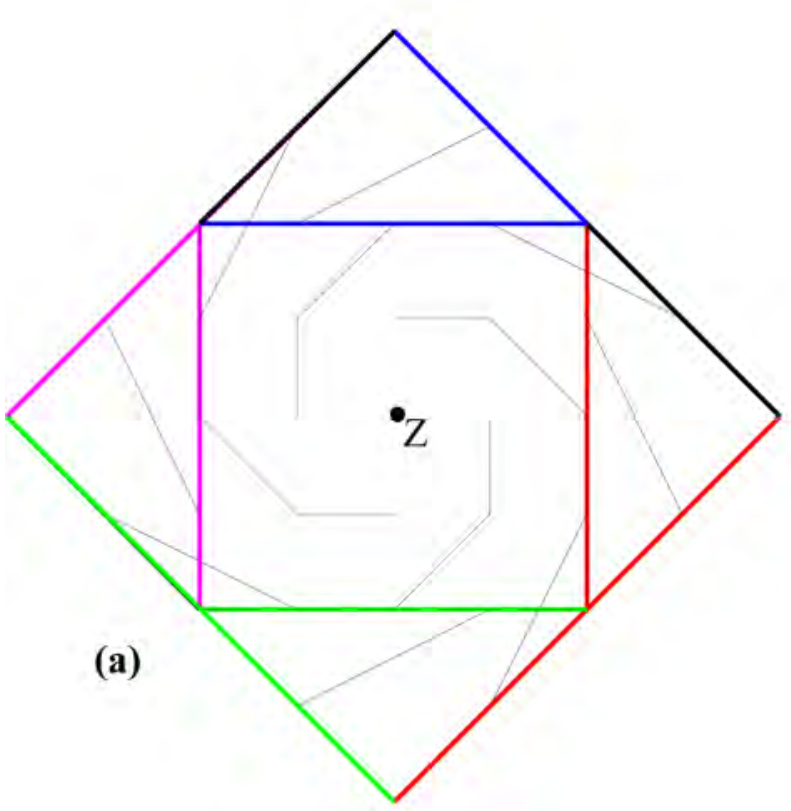

(b)

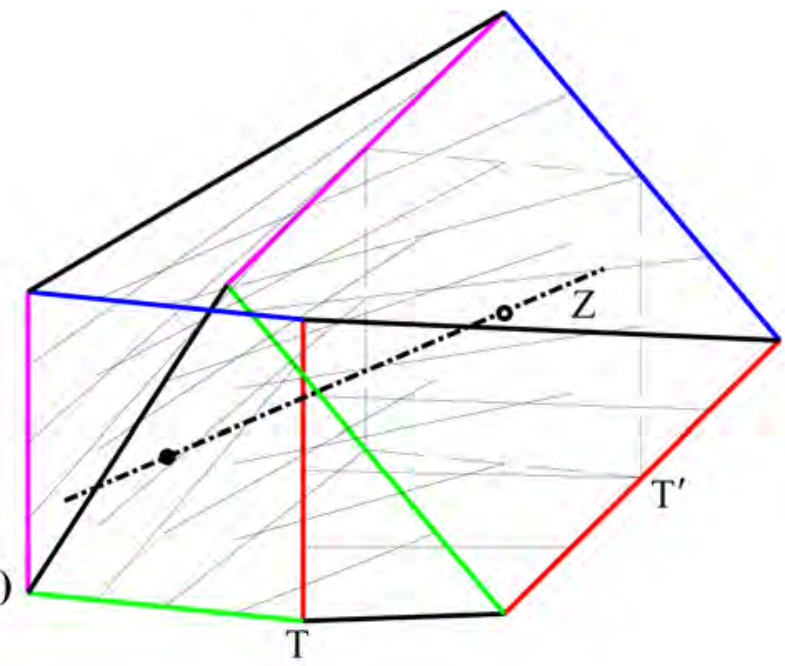

Figure 3 A pencil of divergency $\mathbf{M}=\mathbf{L} / 2 \mathrm{D}$ at entrance plane T. There are no principal meridional planes and no foci. (b) azimuth $-64^{\circ}$, elevation $12^{\circ}$.

\section{Antiscalar divergency}

For an antiscalar divergency the scalar coefficient $M_{\text {I }}$ is zero. Figures 4, 5 and 6 show pencils with antiscalar divergency at entrance plane T; the antisymmetric coefficient $M_{\mathrm{L}}$ increases from zero in Figure 4 to $1 / 4$ D Figure 5 and still further to $1 / 2 \mathrm{D}$ in Figure 6. We choose the ortho-coefficient $M_{\mathrm{J}}$ to be constant at $1 \mathrm{D}$ and the oblique coefficient $M_{\mathrm{K}}$ constant at 0 D. Interchanging the values of $M_{\mathrm{J}}$ and $M_{\mathrm{K}}$ merely has the effect, for an observer upstream of T, of rotating the pencils anticlockwise through $45^{\circ}$. Similarly any other non-zero value of $M_{K}$ merely rotates the pencils.

At transverse plane $\mathrm{T}$ the pencil in Figure 4 has divergency

$\mathbf{M}=\mathbf{J}=\left(\begin{array}{cc}1 & 0 \\ 0 & -1\end{array}\right)$ D.

The discriminant (Equation 4) of the divergency is 4 $\mathrm{D}^{2}$. By Equation 5 there are two distinct principal divergencies, $M_{ \pm}= \pm 1 \mathrm{D}$. The corresponding principal meridional planes are at angles $A_{ \pm}$given by Equations 6, 7 or 8. Equation 6 does not apply because the 
denominator of the fraction on the right-hand side is zero. Equation 7 applies in the case $A_{+}$but not in the case of $A_{-}$. For $A_{-}$one is left with Equation 8. Thus we find that $A_{+}=0^{\circ}$ (shown as 180 in the principal meridional representation of divergency in Table 1) and $A_{-}=90^{\circ}$. The two principal meridional planes are shown by means of dotted lines in Figure 4(b); and in transverse plane $\mathrm{T}$ they define a divergence cross as indicated with the labels 1 and -1 . From Equation 10 we find that the two focal planes are located at $\zeta_{ \pm}=\mp 1 \mathrm{~m}$, that is, $1 \mathrm{~m}$ upstream and $1 \mathrm{~m}$ downstream from T. They are shown in Figure 4(b). The orientations of the two focal lines are given by Equation 11: $\phi_{+}=90^{\circ}$ and $\phi_{-}=0^{\circ}$. The vertical focal line represents focus in the horizontal meridional plane and vice versa. The focal lines are shown as thicker dashed lines labelled $F_{+}$and $F_{\text {. }}$ in Figure 4(a) and (b). At exit plane $\mathrm{T}^{\prime}$ the divergency is

$\mathbf{M}^{\prime}=\frac{2}{3} \mathbf{I}-\frac{1}{3} \mathbf{J}=\left(\begin{array}{cc}1 / 3 & 0 \\ 0 & 1\end{array}\right)$ D.

The pencil is of the conventional type in familiar optical systems; the two focal lines define the interval of Sturm.

We note that the pencil, square in entrance plane $\mathrm{T}$, has been stretched in the horizontal in exit plane $\mathrm{T}^{\prime}$. It has also been turned upside-down; the green line is now on top.

At $\mathrm{T}$ the divergency of the pencil in Figure 5 has an additional antisymmetric component: the divergency is

$$
\mathbf{M}=\mathbf{J}+\frac{1}{4} \mathbf{L}=\left(\begin{array}{cc}
1 & 1 / 4 \\
-1 / 4 & -1
\end{array}\right) \text { D. }
$$

Thus, by Equation 4, $\operatorname{dis} \mathbf{M}=15 / 4 \mathrm{D}^{2}$ and, by Equation 5, the two principal divergencies are $M_{ \pm}= \pm \sqrt{15} / 4$ or about \pm 0.97 D. Equation 6 gives the angles at which the corresponding principal meridional planes lie:

$$
A_{ \pm}=\tan ^{-1} \frac{ \pm \sqrt{15} / 4-1}{1 / 4},
$$

that is, $A_{+}=172.8^{\circ}$ and $A_{-}=97.2^{\circ}$. The focal planes are at $\zeta_{ \pm}=\mp 1.03 \mathrm{~m}$ and the focal lines $\mathrm{F}_{+}$and $\mathrm{F}_{-}$ are at angles $\phi_{+}=97.2^{\circ}$ and $\phi_{-}=172.8^{\circ}$ approximate- ly. The divergency at transverse plane $T^{\prime}$ is

$\mathbf{M}^{\prime}=0.6818 \mathbf{I}-0.3636 \mathbf{J}-0.0909 \mathbf{L}=\left(\begin{array}{cc}0.3182 & -0.0909 \\ 0.0909 & 1.0455\end{array}\right) \mathrm{D}$.

At $\mathrm{T}^{\prime}$ the pencil is a parallelogram. Addition of the antisymmetric component to the divergency has caused the pencil to lose its rectangular shape.

Figure 6 shows the effect of increasing the antisymmetric component of divergency further. At $\mathrm{T}$ the divergency is

$\mathbf{M}=\mathbf{J}+\frac{1}{2} \mathbf{L}=\left(\begin{array}{cc}1 & 1 / 2 \\ -1 / 2 & -1\end{array}\right)$ D.

Hence $\operatorname{dis} \mathbf{M}=3 \mathrm{D}^{2}$, the two principal divergencies are $M_{ \pm}= \pm \sqrt{3} / 2 \mathrm{D}$ and the corresponding principal planes are at

$A_{ \pm}=\tan ^{-1} \frac{ \pm \sqrt{3} / 2-1}{1 / 2}$

by Equation 6. Hence $A_{+}=165^{\circ}$ and $A_{-}=105^{\circ}$, $\zeta_{ \pm}=\mp 2 / \sqrt{3}$ or $\mp 1.15 \mathrm{~m}$ approximately. The focal lines are at $\phi_{+}=105^{\circ}$ and $\phi_{-}=165^{\circ}$. At $\mathrm{T}^{\prime}$ the divergency of the pencil is

$$
\mathbf{M}^{\prime}=\frac{3}{4} \mathbf{I}-\frac{1}{2} \mathbf{J}-\frac{1}{4} \mathbf{L}=\left(\begin{array}{cc}
1 / 4 & -1 / 4 \\
1 / 4 & 5 / 4
\end{array}\right) \mathrm{D} .
$$

With no antisymmetric component the principal meridional planes and focal lines of the pencil in Figure 4 are orthogonal. As the antisymmetric component increases in Figures 5 and 6 the principal meridional planes close scissors-like and symmetrically towards the plane at $135^{\circ}$. The focal lines exhibit the same scissors-like behaviour; and the focal planes move apart.

If the sign of the antisymmetric component is reversed the principal meridional planes and line foci close symmetrically towards $45^{\circ}$ instead while the focal planes move apart. 

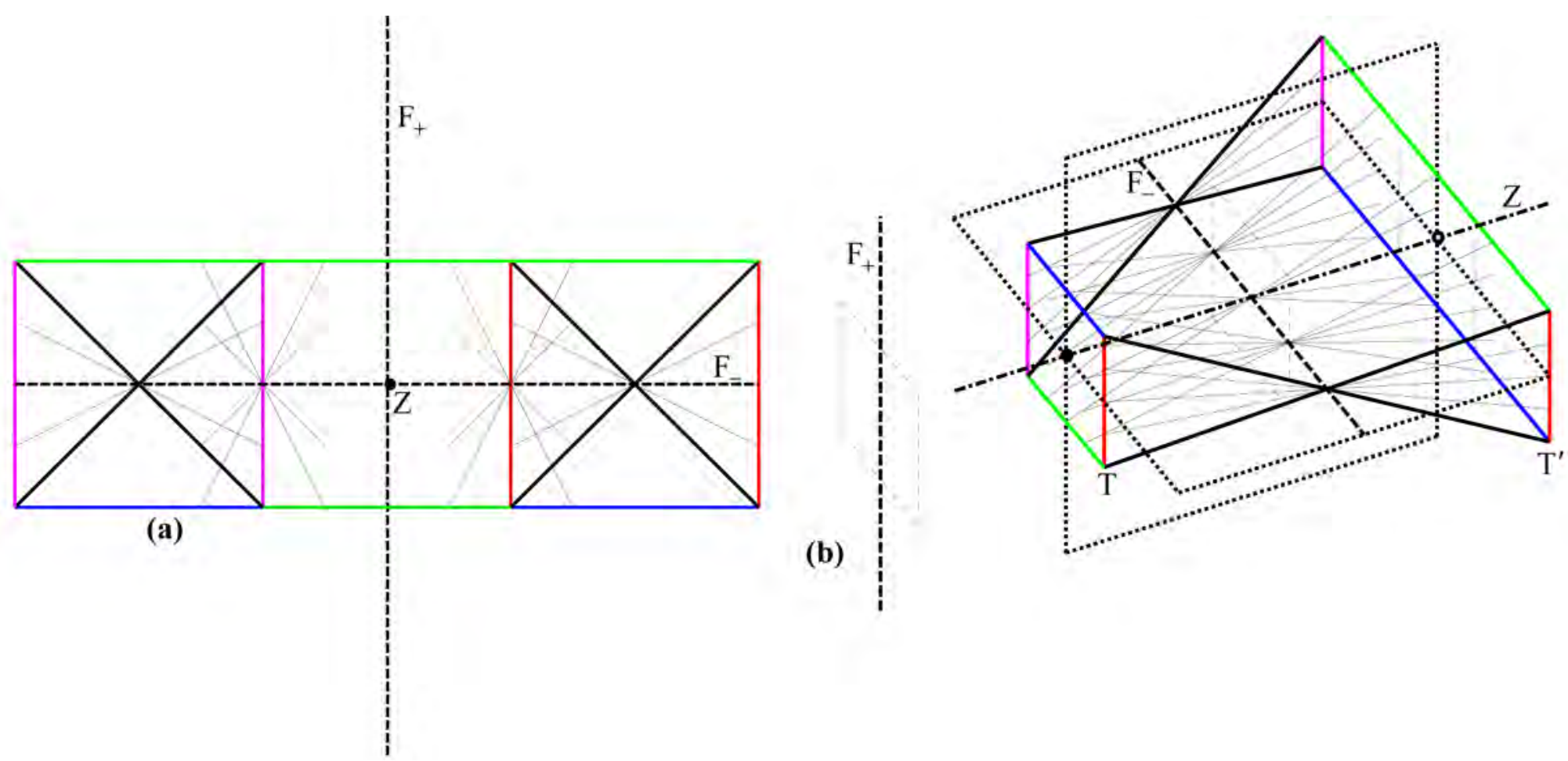

Figure 4 A pencil with divergency $\mathbf{M}=\mathbf{J} \mathrm{D}$ at $\mathrm{T}$. The orthogonal pair of principal meridional planes is shown by means of dotted lines. Light dashed lines define the two focal planes. Heavy dashed lines represent the two orthogonal line foci $F_{+}$and $F_{-}$. One focal plane is upstream from entrance plane $\mathrm{T}$. The rays diverge horizontally and converge vertically from $\mathrm{T}$. The pencil is one possible in conventional astigmatic optical systems; the two focal planes define the interval of Sturm. (b) azimuth $-27^{\circ}$, elevation $38^{\circ}$.
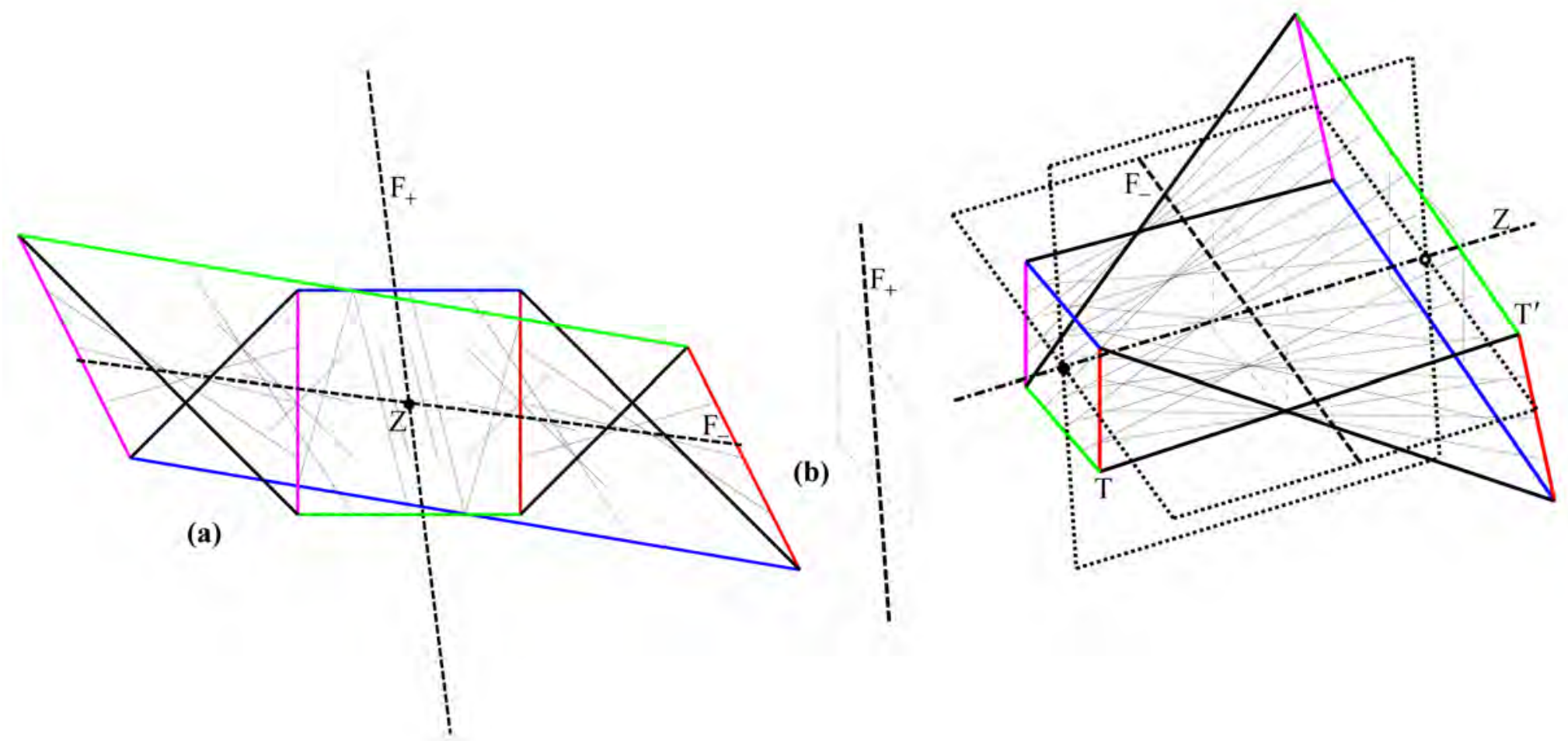

Figure 5 A pencil with divergency $\mathbf{M}=\mathbf{J}+\mathbf{L} / 4 \mathrm{D}$ at $\mathrm{T}$. The antisymmetric contribution to $\mathbf{M}$ causes the two principal meridional planes and line foci to rotate towards each other and an meridional plane at $135^{\circ}$. Relative to their positions in Figure 4 the focal planes have moved further apart. (b) azimuth $-27^{\circ}$, elevation $38^{\circ}$. 

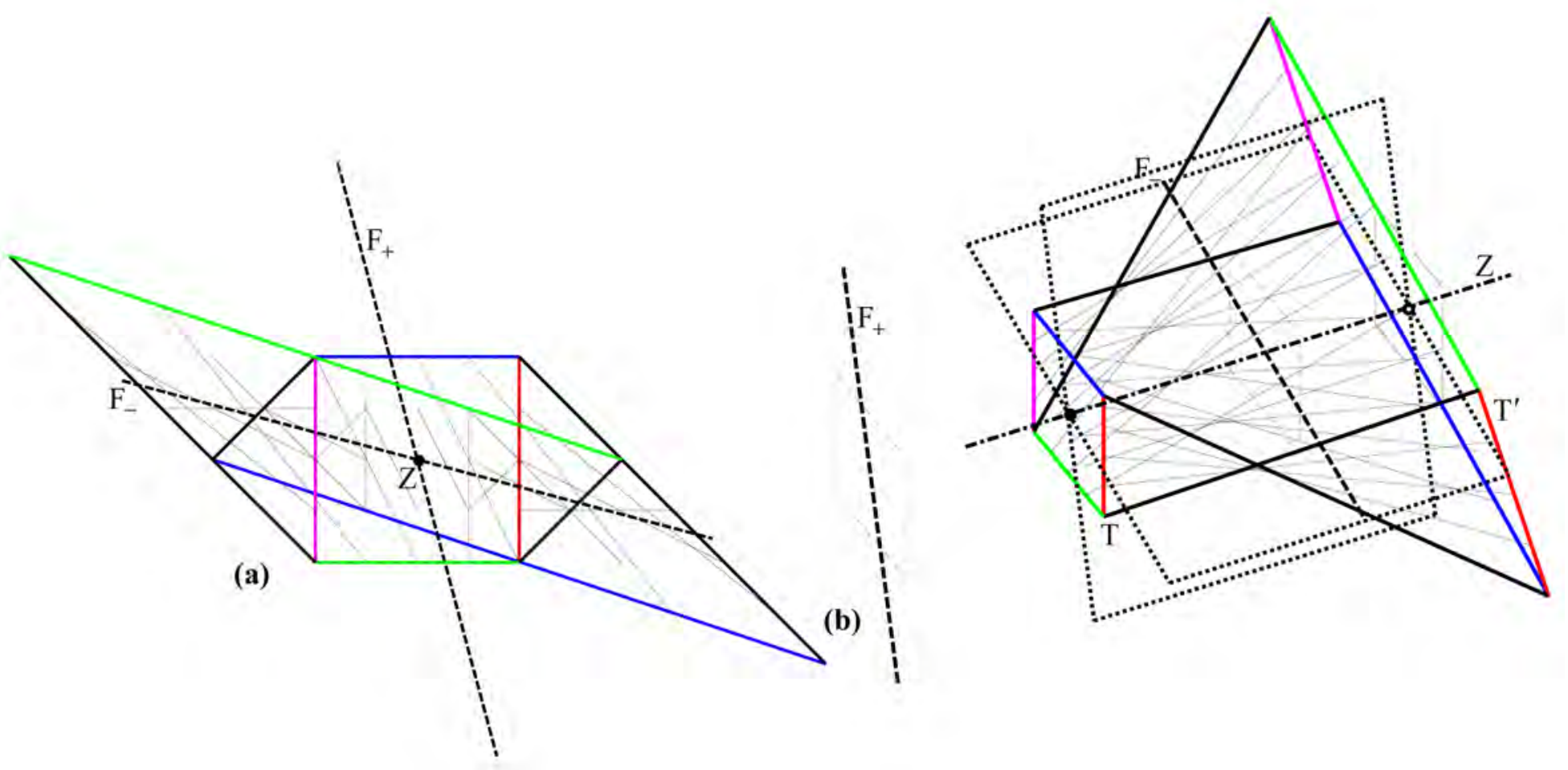

Figure 6 A pencil with divergency $\mathbf{M}=\mathbf{J}+\mathbf{L} / 2 \mathrm{D}$ at $\mathrm{T}$. The rotation of the principal meridional planes and the rotation and displacement of the focal lines evident in Figure 5 contines here. (b) azimuth $-27^{\circ}$, elevation $38^{\circ}$.

\section{Scalar divergency with an added antisymmetric component}

Figure 7 represents a pencil of scalar divergency. Figures 8 and 9 show the effect of adding an antisymmetric component to the divergency.

The divergency at $\mathrm{T}$ in Figure 7 is

$$
\mathbf{M}=-\mathbf{I} \text { D. }
$$

It is a negative scalar divergency and the rays in the pencil converge towards a focal point. The focal point $\mathrm{F}$ is at $1 \mathrm{~m}$. Every meridional plane is a principal meridional plane. At $\mathrm{T}^{\prime}$ the divergency of the pencil is $\mathbf{M}^{\prime}=\mathbf{I} \mathrm{D}$.

The pencil is of the familiar non-astigmatic type.

We now add $\mathbf{L}$ / 4 D to the divergency of the pencil at $\mathrm{T}$ in Figure 7 . The result is the divergency

$$
\mathbf{M}=-\mathbf{I}+\frac{1}{4} \mathbf{L}=\left(\begin{array}{cc}
-1 & 1 / 4 \\
-1 / 4 & -1
\end{array}\right) \mathrm{D} \text {. }
$$

The pencil is shown in Figure 8. The discriminant is negative. Hence there are no principal meridional planes or foci. The divergency at $\mathrm{T}^{\prime}$ is
$\mathbf{M}^{\prime}=\frac{9}{10} \mathbf{I}+\frac{1}{5} \mathbf{L}=\left(\begin{array}{cc}9 / 10 & 1 / 5 \\ -1 / 5 & 9 / 10\end{array}\right)$ D.

Adding an additional L / 4 D we obtain the divergency

$\mathbf{M}=-\mathbf{I}+\frac{1}{2} \mathbf{L}=\left(\begin{array}{cc}-1 & 1 / 2 \\ -1 / 2 & -1\end{array}\right) \mathrm{D}$

of the pencil at transverse plane $\mathrm{T}$. The pencil is shown in Figure 9. Again there are no principal meridional planes or foci and the emergent divergency at $\mathrm{T}^{\prime}$ is

$$
\mathbf{M}^{\prime}=\frac{3}{4} \mathbf{I}+\frac{1}{4} \mathbf{L}=\left(\begin{array}{cc}
3 / 4 & 1 / 4 \\
-1 / 4 & 3 / 4
\end{array}\right) \text { D. }
$$

We see from the cases represented by Figures 7, 8 and 9 that the anstisymmetric component of the divergency obliterates the point focus and enlarges it into a neck. As for the sequence Figures 1 to 3 the antisymmetric component induces chirality; it converts the achiral pencil in Figure 7 to the chiral pencils in Figures 8 and 9. Rotational invariance about $\mathrm{Z}$ is preserved. 
(a)

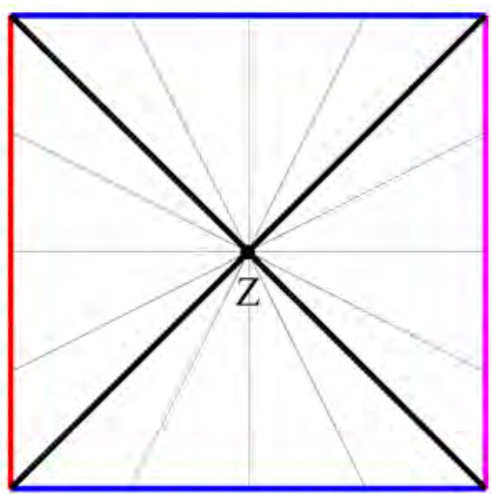

(b)

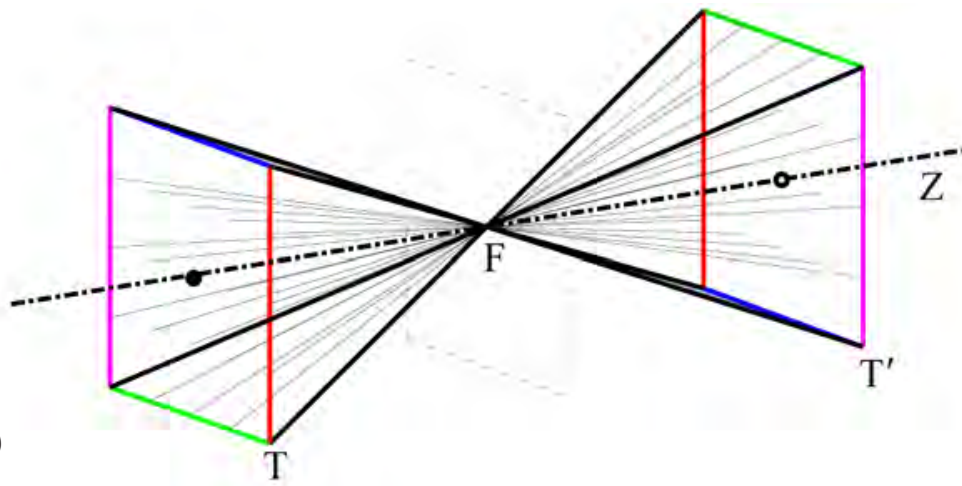

Figure 7 A pencil with divergency $\mathbf{M}=-\mathbf{I} \mathrm{D}$ at $\mathrm{T}$. The pencil is conventional with focal plane and point focus. All meridional planes are principal meridional planes. (b) azimuth $-34^{\circ}$, elevation $14^{\circ}$.

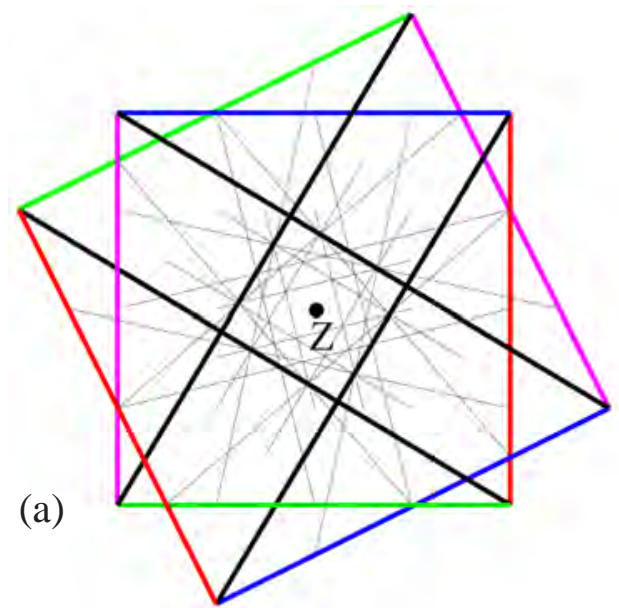

(b)

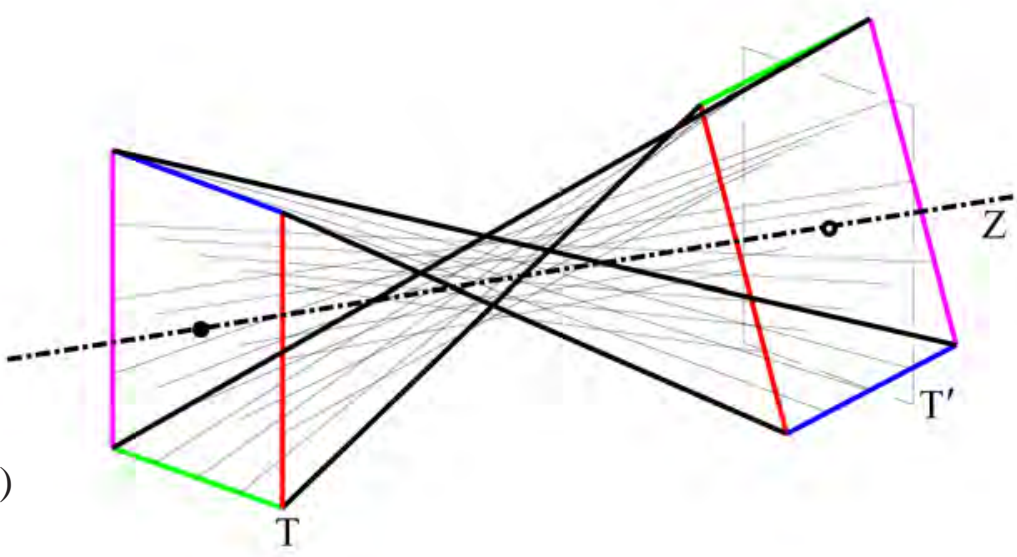

Figure 8 A pencil with divergency $\mathbf{M}=-\mathbf{I}+\mathbf{L} / 4 \mathrm{D}$ at $\mathrm{T}$. The addition of the antistigmatic contribution has caused the rays to separate at the point focus in Figure 7 . There is no focus and there are no principal meridional planes. (b) azimuth $-34^{\circ}$, elevation $14^{\circ}$.
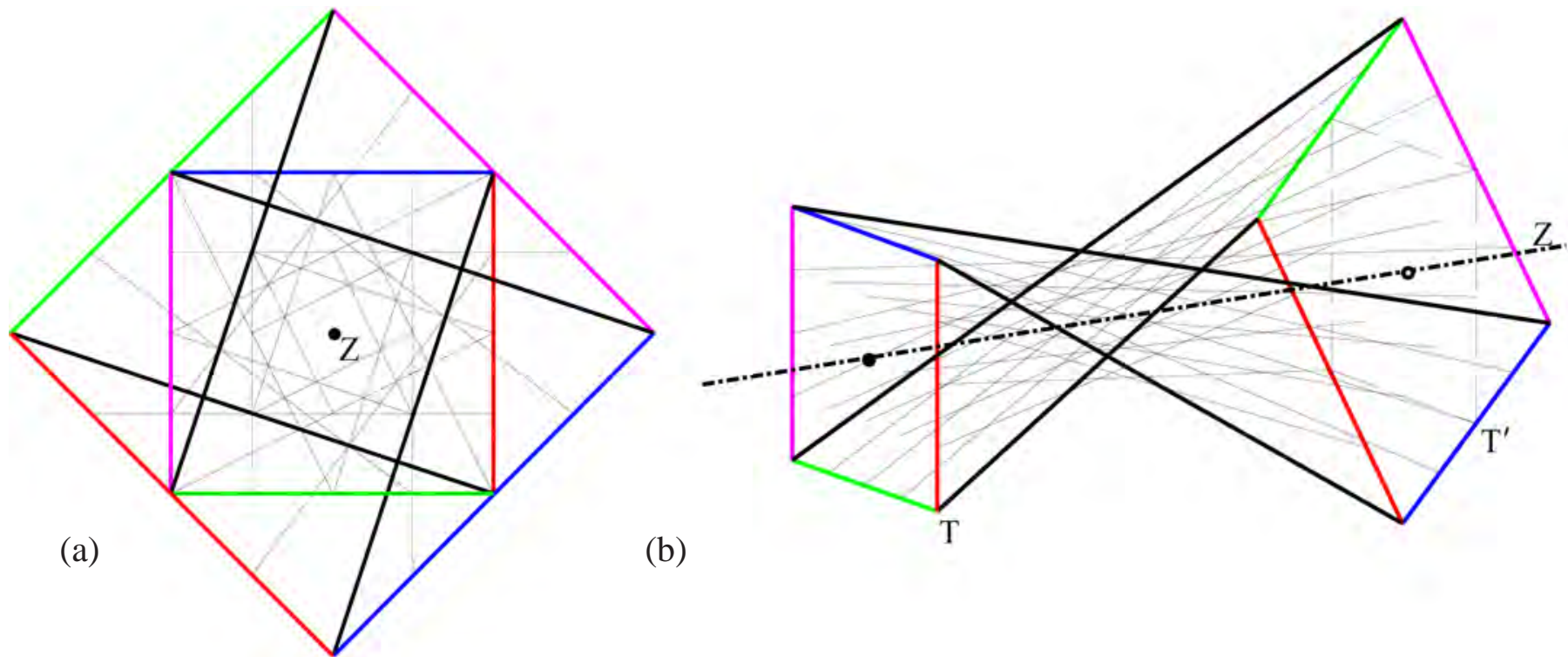

(b)

Figure 9 A pencil with divergency $\mathbf{M}=-\mathbf{I}+\mathbf{L} / 2 \mathrm{D}$ at T. There are no principal meridional planes or foci. (b) azimuth $-34^{\circ}$, elevation $14^{\circ}$. 
Symmetric divergency with added antisymmetric component

In Figures 10, 11 and 12 we examine the effect of adding an antisymmetric component to a symmetric non-scalar divergency.

Figure 10 represents a pencil with divergency

$\mathbf{M}=-\mathbf{I}+\mathbf{J} / 4=\left(\begin{array}{cc}-3 / 4 & 0 \\ 0 & -5 / 4\end{array}\right) \mathrm{D}$

at T. Thus $\operatorname{dis} \mathbf{M}=1 / 4 \mathrm{D}^{2}$ and $M_{+}=-3 / 4 \mathrm{D}$ and $M_{-}=-5 / 4$ D. Equation 6 does not apply. Equation 7 gives $A_{+}=0^{\circ}$ and Equation 8 gives $A_{-}=90^{\circ}$. Then $\zeta_{+}=4 / 3 \mathrm{~m}$ and $\zeta_{-}=4 / 5 \mathrm{~m}$. Finally the focal lines are at $\phi_{+}=90^{\circ}$ and $\phi_{-}=0^{\circ}$. At $\mathrm{T}^{\prime}$ $\mathbf{M}^{\prime}=\frac{7}{6} \mathbf{I}+\frac{1}{3} \mathbf{J}=\left(\begin{array}{cc}3 / 2 & 0 \\ 0 & 5 / 6\end{array}\right)$ D.

The pencil is an example of the familiar type of astigmatic pencil with associated orthogonal line foci and interval of Sturm.

Adding $\mathbf{L} / 4 \mathrm{D}$ to the divergency of the pencil at $\mathrm{T}$ in Figure 10 we obtain the pencil shown in Figure 11. The incident divergency is

$\mathbf{M}=-\mathbf{I}+\frac{1}{4} \mathbf{J}+\frac{1}{4} \mathbf{L}=\left(\begin{array}{cc}-3 / 4 & 1 / 4 \\ -1 / 4 & -5 / 4\end{array}\right)$ D.

Here $\operatorname{dis} \mathbf{M}=0 \quad \mathrm{D}^{2}$ and it follows that there is a unique principal divergency given by Equation $5, M=-1 \mathrm{D}$. The principal meridional plane is at (Equation 6) $A=135^{\circ}$. (It bisects the right-angle formed by the principal meridional planes in Figure 10.) The focal plane is at $\zeta=1 \mathrm{D}$ and the unique focal line $\mathrm{F}$ is at $\phi=135^{\circ}$. At exit plane $\mathrm{T}^{\prime}$

$\mathbf{M}^{\prime}=\mathbf{I}+\frac{1}{4} \mathbf{J}+\frac{1}{4} \mathbf{L}=\left(\begin{array}{cc}5 / 4 & 1 / 4 \\ -1 / 4 & 3 / 4\end{array}\right) \mathbf{D}$.

When an additional component $\mathbf{L}$ / $4 \mathrm{D}$ is added the divergency at $\mathrm{T}$ becomes

$\mathbf{M}=-\mathbf{I}+\frac{1}{4} \mathbf{J}+\frac{1}{2} \mathbf{L}=\left(\begin{array}{cc}-3 / 4 & 1 / 2 \\ -1 / 2 & -5 / 4\end{array}\right)$ D.

and the pencil appears as in Figure 12. The discrimi- nant is now negative and there are no principal divergencies, principal planes or foci. At $\mathrm{T}^{\prime}$

$\mathbf{M}^{\prime}=0.7857 \mathbf{I}+0.1429 \mathbf{J}+0.2857 \mathbf{L}=\left(\begin{array}{cc}0.9286 & 0.2857 \\ -0.2857 & 0.6429\end{array}\right)$

D.

Here the addition of an antisymmetric component brings the two focal planes together and the two focal lines $F_{+}$and $F_{-}$close to a single focal line $F$ at $135^{\circ}$ in Figure 11 which disappears in Figure 12.

Figures 13 to 15 repeat Figures 10 to 12 except that the ortho-component of the divergency has been doubled. Again the antisymmetric component brings the two focal planes together and reduces the two focal lines to one focal line. At $\mathrm{T}$ in Figure 13 the divergency is

$\mathbf{M}=-\mathbf{I}+\frac{1}{2} \mathbf{J}=\left(\begin{array}{cc}-1 / 2 & 0 \\ 0 & -3 / 2\end{array}\right)$ D.

The principal divergencies are $M_{+}=-1 / 2 \mathrm{D}$ and $M_{-}=-3 / 2 \mathrm{D}$ and the corresponding principal meridional planes are horizontal (Equation 7) and vertical (Equation 8) respectively. The corresponding focal planes are located at 2 and 2/3 m respectively and the focal lines in them are vertical and horizontal respectively.

At $\mathrm{T}$ in Figure 14 the divergency is

$\mathbf{M}=-\mathbf{I}+\frac{1}{2} \mathbf{J}+\frac{1}{4} \mathbf{L}=\left(\begin{array}{cc}-1 / 2 & 1 / 4 \\ -1 / 4 & -3 / 2\end{array}\right) \mathrm{D}$

which has principal divergencies

$M_{ \pm}=-1 \pm \sqrt{3} / 4 \mathrm{D}$,

that is, $M_{+}=-0.5670 \mathrm{D}$ and $M_{-}=-1.4330 \mathrm{D}$. The corresponding principal meridional planes are at

$$
A_{ \pm}=\tan ^{-1} \frac{M_{ \pm}+1 / 2}{1 / 4}
$$

or $A_{+}=165^{\circ}$ and $A_{-}=105^{\circ}$. Also $\zeta_{+}=1.764 \mathrm{~m}$, $\zeta_{-}=0.698 \mathrm{~m}, \phi_{+}=105^{\circ}$ and $\phi_{-}=165^{\circ}$.

The divergency at $\mathrm{T}$ in Figure 15 is

$$
\mathbf{M}=-\mathbf{I}+\frac{1}{2} \mathbf{J}+\frac{1}{2} \mathbf{L}=\left(\begin{array}{cc}
-1 / 2 & 1 / 2 \\
-1 / 2 & -3 / 2
\end{array}\right) \mathbf{D}
$$


Because the discriminant is now zero there is a single principal divergency, $-1 \mathrm{D}$. The single principal meridional plane is at $135^{\circ}$ and the single focal plane is located at $1 \mathrm{~m}$. It contains the single focal line.

A comment may be worth making about the vertical focal line in the exit plane in Figure 13. In this case $\mathbf{I}+\zeta \mathbf{M}$ and $\mathbf{M}^{-1}+\zeta \mathbf{I}$ are both singular at $\mathrm{T}^{\prime}$. Thus neither Equation 2 nor Equation 3 applies and the divergency $\mathbf{M}^{\prime}$ at $\mathrm{T}^{\prime}$ is undefined. Informally, however, we have

$$
\mathbf{M}^{\prime}=\left(\begin{array}{cc}
-1 / 2 & 0 \\
0 & -3 / 2
\end{array}\right)\left(\begin{array}{cc}
0 & 0 \\
0 & -2
\end{array}\right)^{-1}
$$

from Equation 2. Hence

$$
\mathbf{M}^{\prime}=\left(\begin{array}{cc}
-1 / 2 & 0 \\
0 & -3 / 2
\end{array}\right)\left(\begin{array}{cc}
\infty & 0 \\
0 & -1 / 2
\end{array}\right)=\left(\begin{array}{cc}
\infty & 0 \\
0 & 3 / 4
\end{array}\right)
$$

or

$$
\mathbf{M}^{\prime}=\left(\begin{array}{cc}
\infty & 0 \\
0 & 3 / 4
\end{array}\right) \text { D. }
$$

Thus the divergency is infinite in the horizontal meridian but finite in the vertical meridian. Expanded in terms of components the divergency at $T^{\prime}$ is $\mathbf{M}^{\prime}=\infty \mathbf{I}+\infty \mathbf{J}$ D.

Notice that information is lost in going from Equation 13 to Equation 14; the matrix on the right of Equation 13 cannot be recovered from Equation 14. An infinite divergency $\mathbf{M}^{\prime}$ occurs wherever a line or point focus lies in the exit plane $\mathrm{T}^{\prime}$.

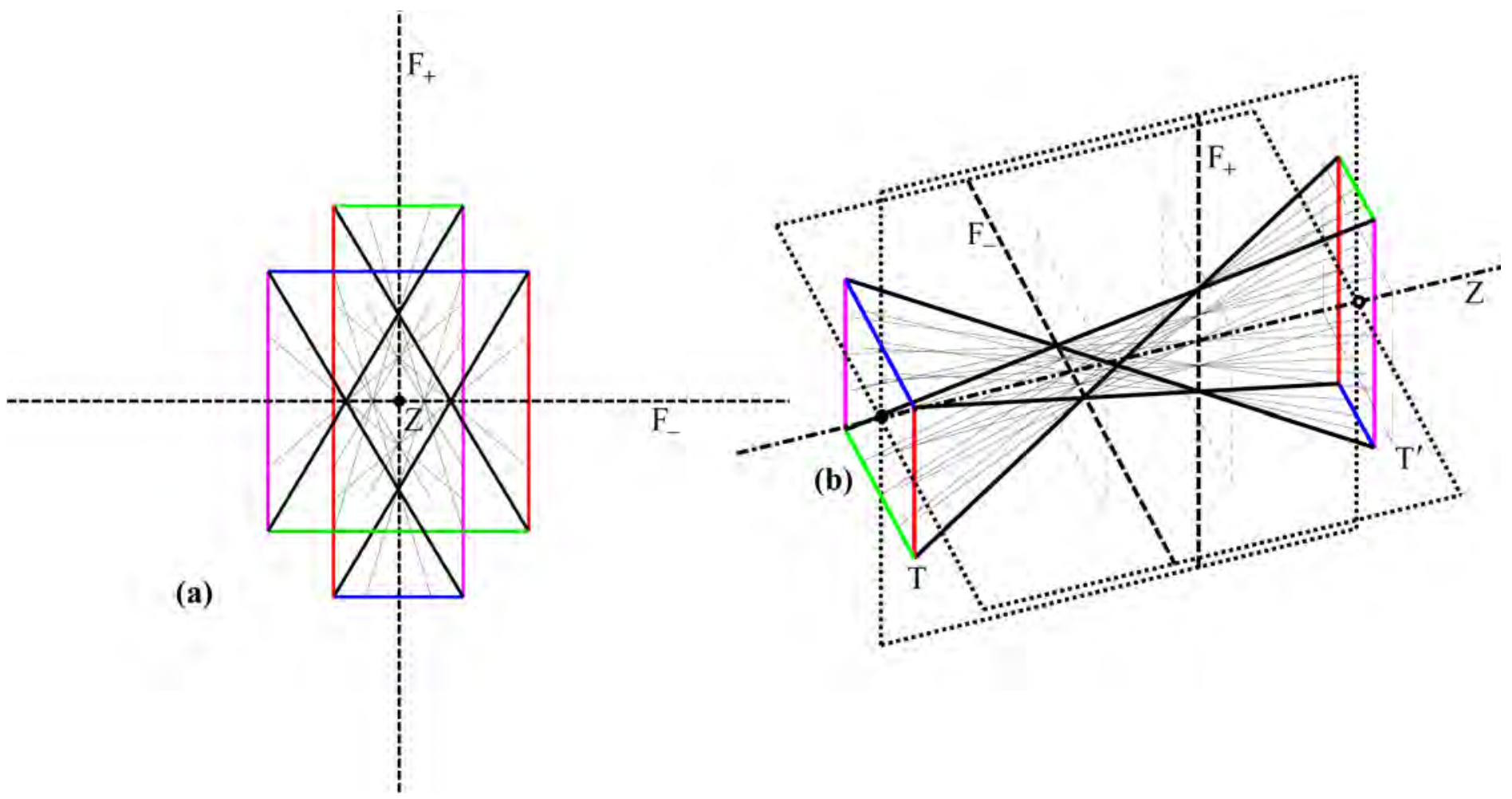

Figure 10 A pencil with divergency $\mathbf{M}=-\mathbf{I}+\mathbf{J} / 4 \mathrm{D}$ at $\mathrm{T}$. The pencil is conventional. The orthogonal line foci define the interval of Sturm. (b) azimuth $-20^{\circ}$, elevation $42^{\circ}$. 

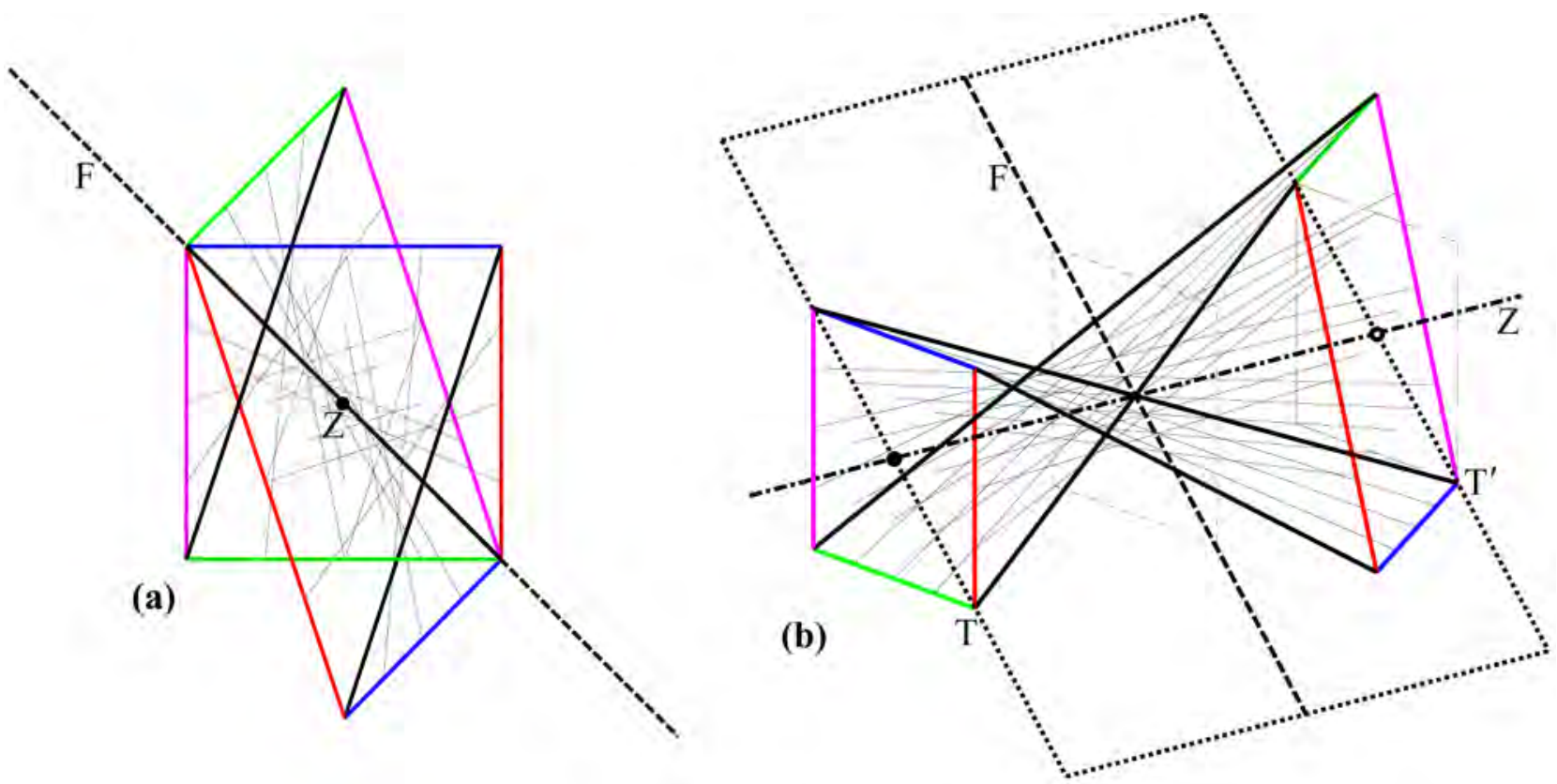

Figure 11 A pencil with divergency $\mathbf{M}=-\mathbf{I}+\mathbf{J} / 4+\mathbf{L} / 4 \mathrm{D}$ at $\mathrm{T}$. The pencil has a single line focus. The antisymmetric component has caused the principal meridional planes to rotate symmetrically towards each other becoming one at $135^{\circ}$ and bisecting the angle between the original principal meridional planes. (b) azimuth $-40^{\circ}$, elevation $18^{\circ}$.

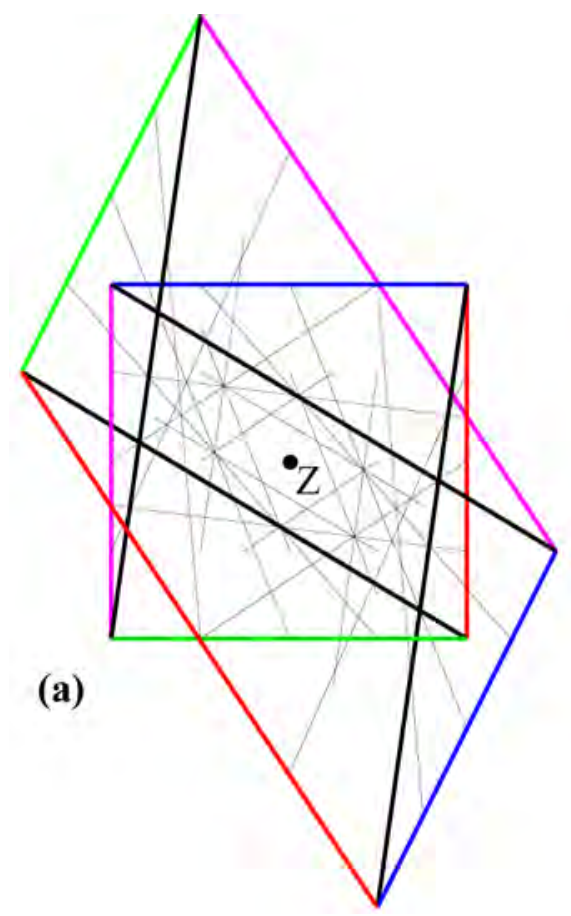

(b)

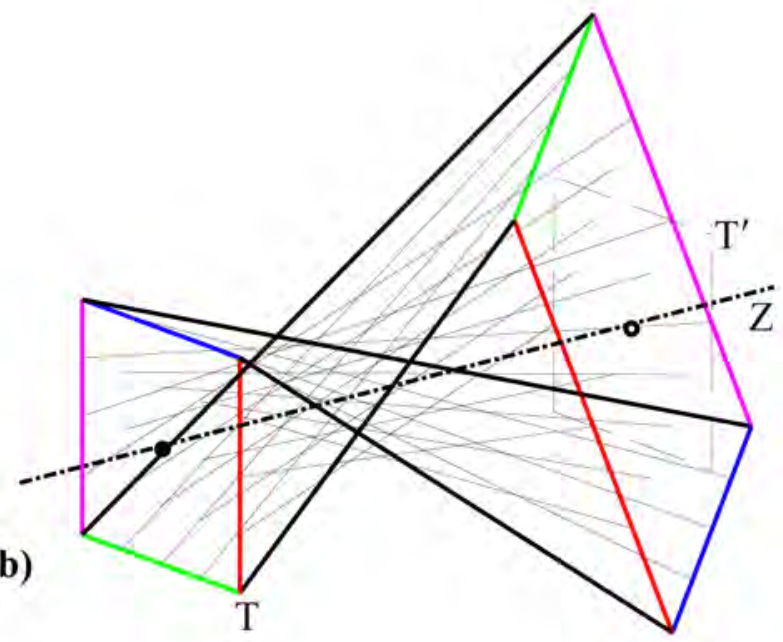

Figure 12 A pencil with divergency $\mathbf{M}=-\mathbf{I}+\mathbf{J} / 4+\mathbf{L} / 2 \mathrm{D}$ at $\mathrm{T}$. There are no principal meridional planes or foci. The additional antisymmetric component has obliterated the single line focus of Figure 11 . (b) azimuth $-40^{\circ}$, elevation $18^{\circ}$. 


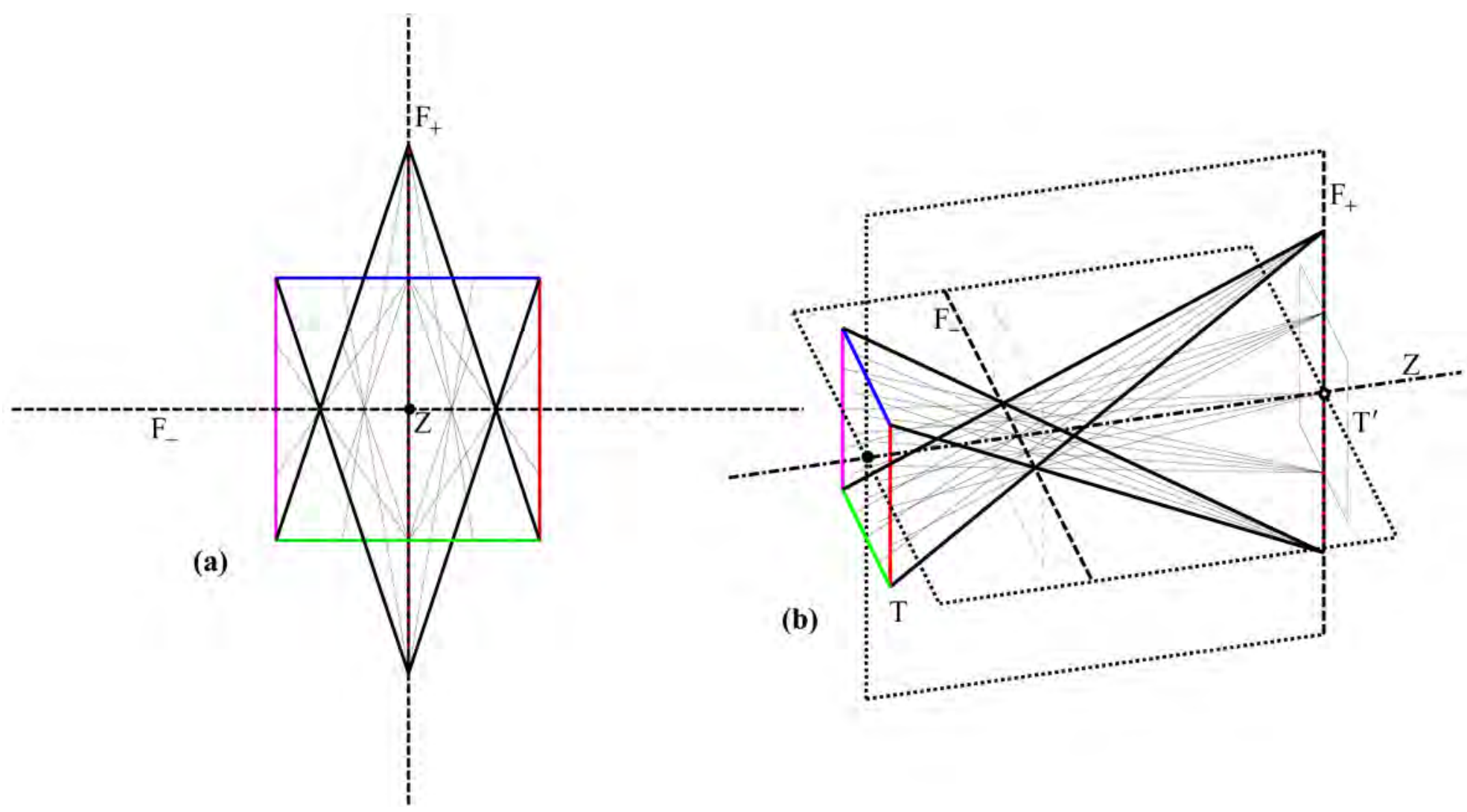

Figure 13 A pencil with divergency $\mathbf{M}=-\mathbf{I}+\mathbf{J} / 2 \mathrm{D}$ at $\mathrm{T}$. The pencil is conventional. The second (vertical) focal line is on exit plane $\mathrm{T}^{\prime}$. (b) azimuth $-15^{\circ}$, elevation $32^{\circ}$.

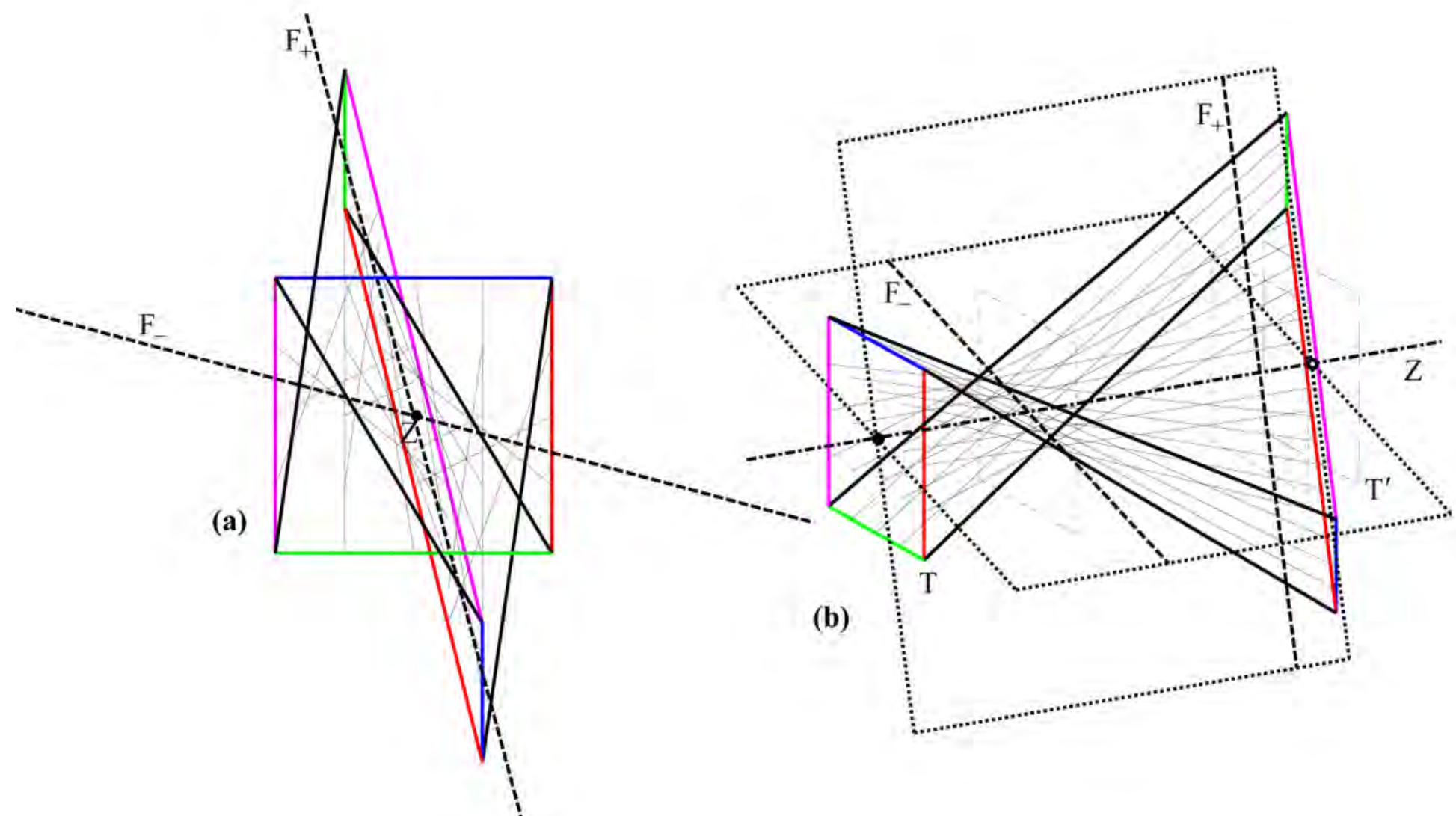

Figure 14 A pencil with divergency $\mathbf{M}=-\mathbf{I}+\mathbf{J} / 2+\mathbf{L} / 4 \mathrm{D}$ at $\mathrm{T}$. The antisymmetric component has caused the line foci to rotate and the focal planes to move towards each other. (b) azimuth $-29^{\circ}$, elevation $18^{\circ}$. 

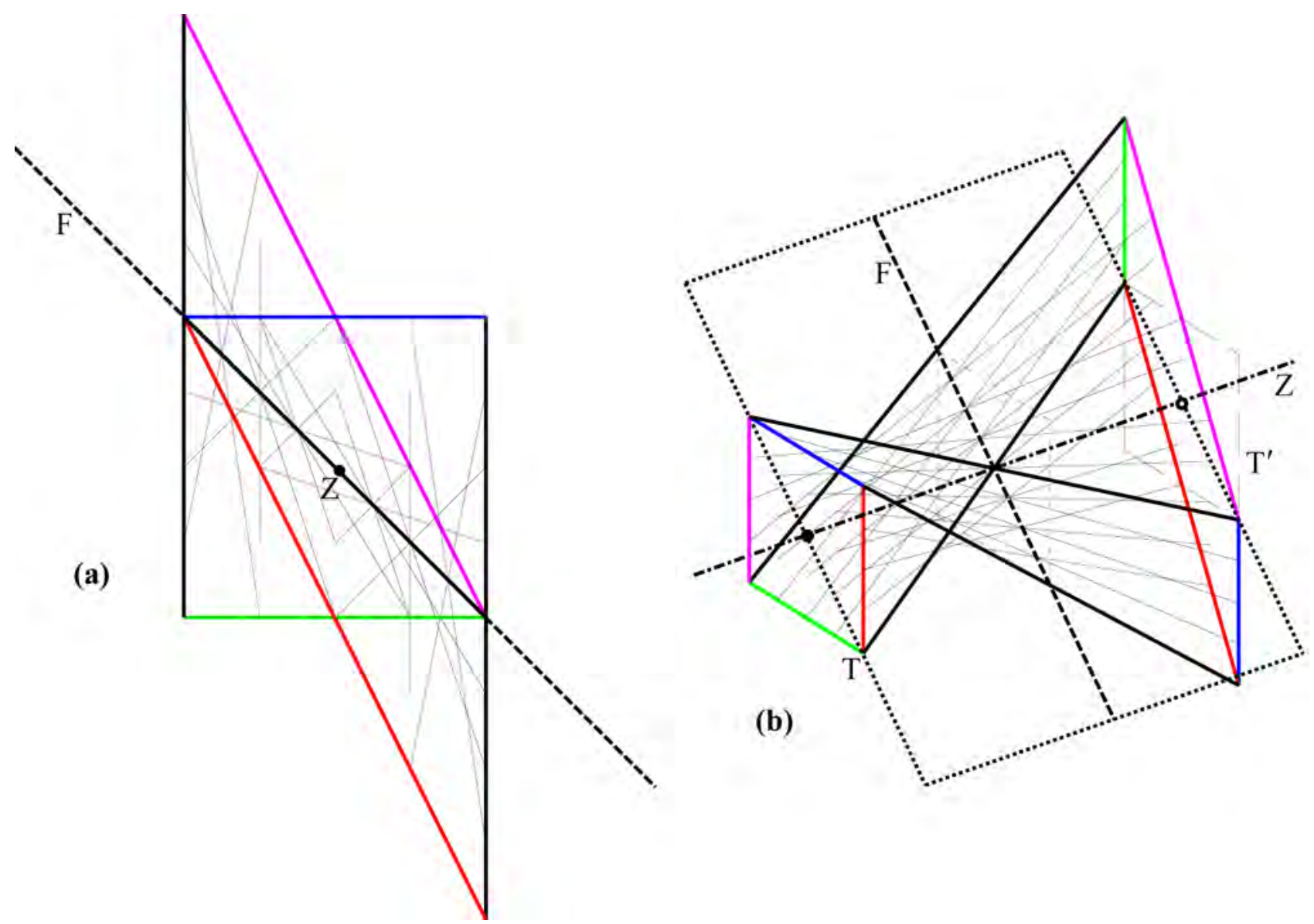

Figure 15 A pencil with divergency $\mathbf{M}=-\mathbf{I}+\mathbf{J} / 2+\mathbf{L} / 2 \mathrm{D}$ at $\mathrm{T}$. There is a single focal line. The focal planes of Figures 13 and 14 have merged into one. (b) azimuth $-29^{\circ}$, elevation $18^{\circ}$.

\section{Singular divergency with added antisymmetric component}

A pencil with a singular divergency $\mathbf{M}$ is shown in Figure 16. The divergency at $\mathrm{T}$ is

$\mathbf{M}=-\mathbf{I}+\mathbf{J}=\left(\begin{array}{cc}0 & 0 \\ 0 & -2\end{array}\right)$ D.

In more conventional terminology one might describe the pencil as cylindrical. The positive discriminant shows there are two principal divergencies: $M_{+}=0 \mathrm{D}$ and $M_{-}=-2 \mathrm{D}$. From Equation $7 A_{+}=0^{\circ}$ and from Equation $8 A_{-}=90^{\circ}$. The focal planes are at infinity and $1 / 2 \mathrm{~m}$ respectively. Only the latter focal plane is shown in Figure 16; it contains the only finite focal line (horizontal).

Figures 17 to 21 shows the effect of adding increasing amounts of the antisymmetric component to the divergency at entrance plane T. In Figure 17 the divergency is
$\mathbf{M}=-\mathbf{I}+\mathbf{J}+\frac{1}{4} \mathbf{L}=\left(\begin{array}{cc}0 & 1 / 4 \\ -1 / 4 & -2\end{array}\right)$ D.

The principal meridional planes have begun rotating towards one another and towards the plane at $135^{\circ}$. The second focal plane (not shown) is now located at a finite reduced distance (about $31.5 \mathrm{~m}$ ). In Figure 18 the divergency at $\mathrm{T}$ is

$$
\mathbf{M}=-\mathbf{I}+\mathbf{J}+\frac{1}{2} \mathbf{L}=\left(\begin{array}{cc}
0 & 1 / 2 \\
-1 / 2 & -2
\end{array}\right) \mathbf{D}
$$

and the rotation and displacement continues. The second focal plane, shown at smaller scale in (c), is at reduced distance $7.5 \mathrm{~m}$ approximately.

The pencil in Figure 19 has divergency

$$
\mathbf{M}=-\mathbf{I}+\mathbf{J}+\frac{3}{4} \mathbf{L}=\left(\begin{array}{cc}
0 & 3 / 4 \\
-3 / 4 & -2
\end{array}\right) \mathrm{D}
$$

at entrance plane $\mathrm{T}$. The second focal plane, at about $2.95 \mathrm{~m}$, is now shown in (b). In Figure 20 the two 
principal meridional planes become a single principal meridional plane at $135^{\circ}$; the pencil has divergency

$\mathbf{M}=-\mathbf{I}+\mathbf{J}+\mathbf{L}=\left(\begin{array}{cc}0 & 1 \\ -1 & -2\end{array}\right) \mathrm{D}$

at T. There is a single focal line F. If the antisymmet- ric component of the divergency is increased further the principal planes disappear as does the line focus. This is seen in Figure 21 where the divergency is

$$
\mathbf{M}=-\mathbf{I}+\mathbf{J}+\frac{5}{4} \mathbf{L}=\left(\begin{array}{cc}
0 & 5 / 4 \\
-5 / 4 & -2
\end{array}\right) \mathrm{D}
$$

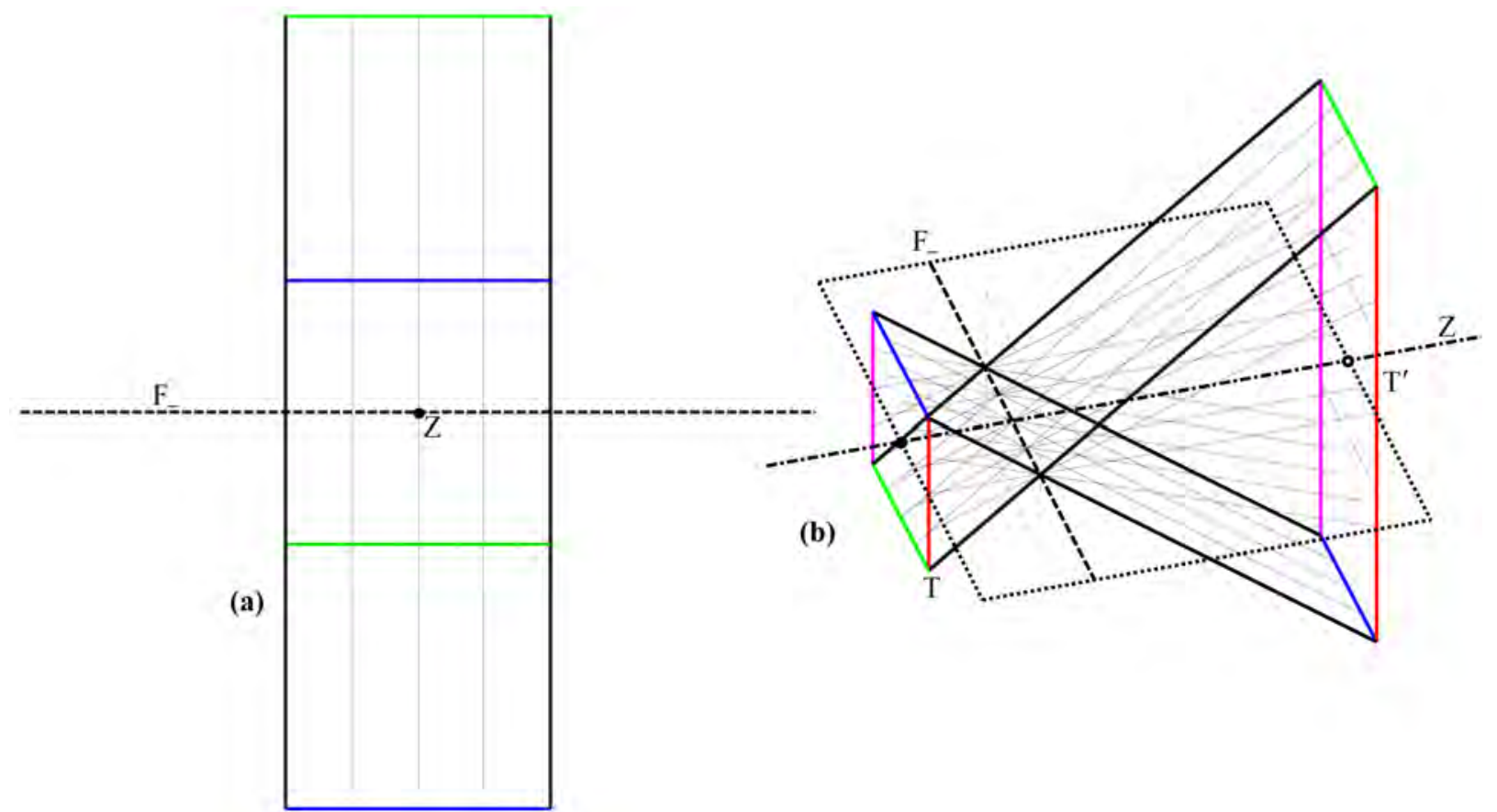

Figure 16 A pencil with divergency $\mathbf{M}=-\mathbf{I}+\mathbf{J} \mathrm{D}$ at $\mathrm{T}$. The divergency is singular. Its (horizontal) line focus is shown. One can regard the other (vertical) line focus as at infinity. (b) azimuth $-17^{\circ}$, elevation $36^{\circ}$.
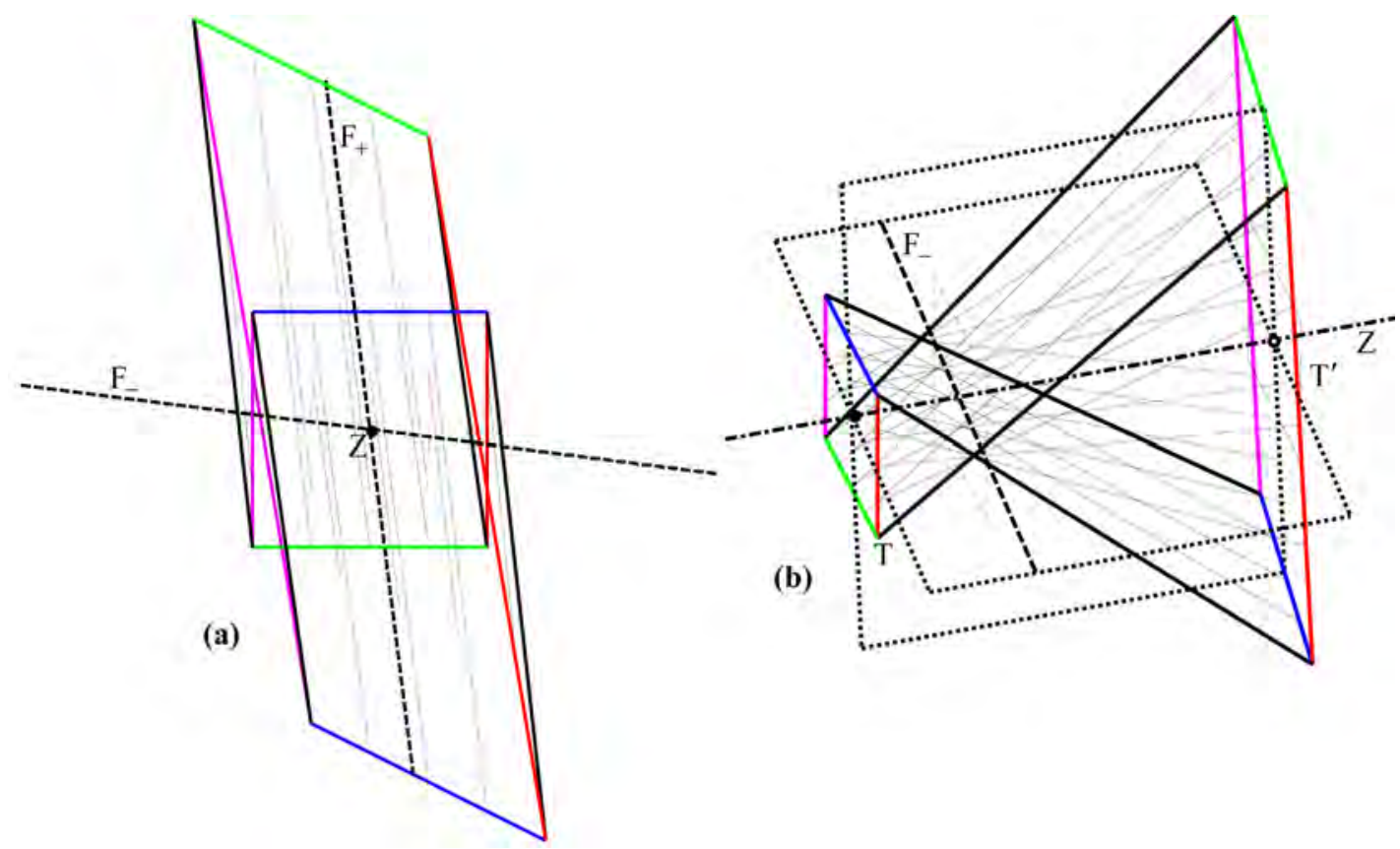

Figure 17 A pencil with divergency $\mathbf{M}=-\mathbf{I}+\mathbf{J}+\mathbf{L} / 4 \mathrm{D}$ at $\mathrm{T}$. The two principal meridional planes are not orthogonal. The focal plane at infinity in Figure 16 is now at a reduced distance of about $32 \mathrm{~m}$. It is not shown. (b) azimuth $-17^{\circ}$, elevation $36^{\circ}$. 

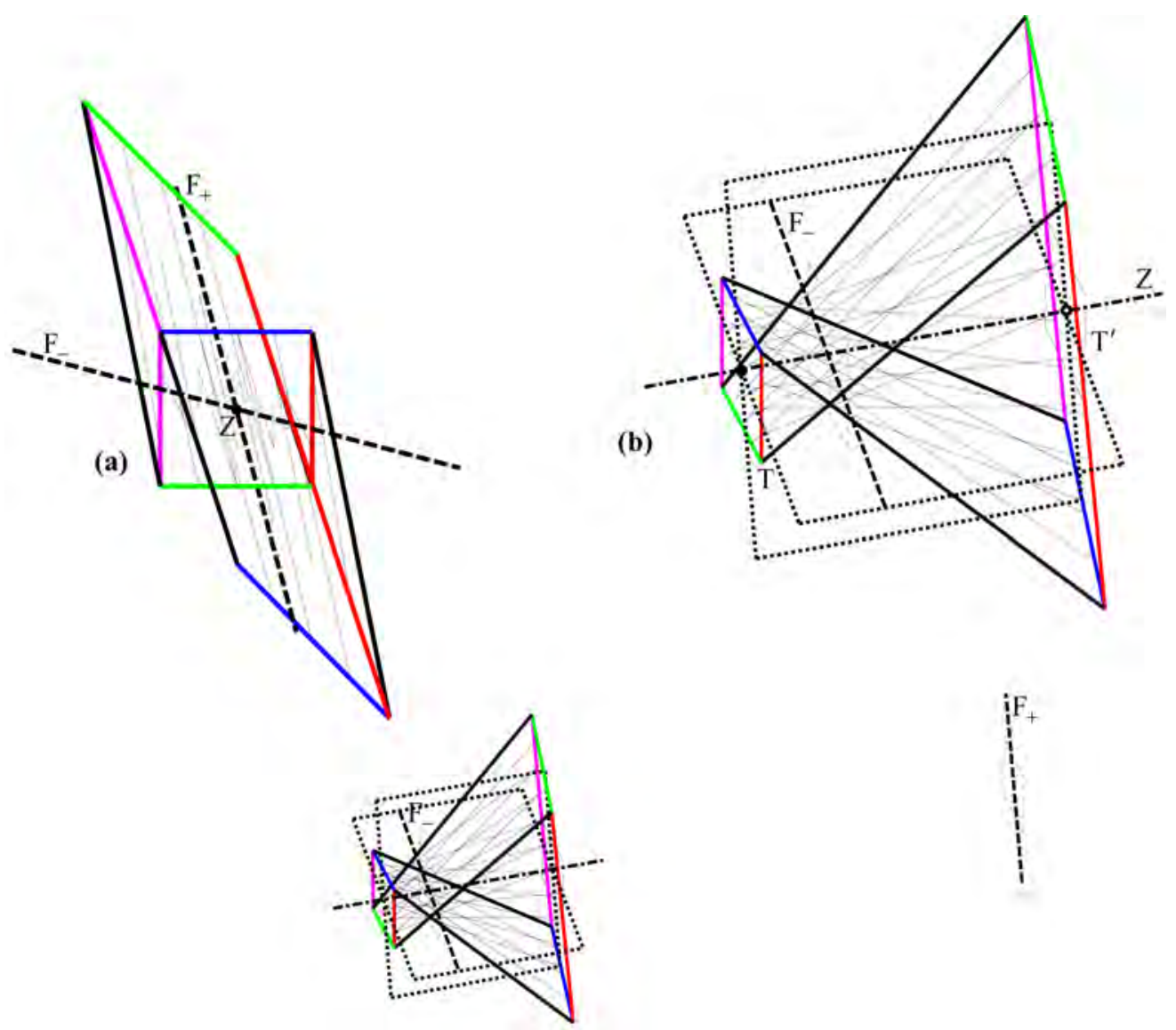

Figure 18 A pencil with divergency $\mathbf{M}=-\mathbf{I}+\mathbf{J}+\mathbf{L} / 2 \mathrm{D}$ at $\mathrm{T}$. Relative to Figure 17 the principal meridional planes have rotated further towards each other and the focal planes continue to approach one another. The second focal plane, shown in (c), is now at a reduced distance of about $7.5 \mathrm{~m}$. (b) azimuth $-17^{\circ}$, elevation $36^{\circ}$.
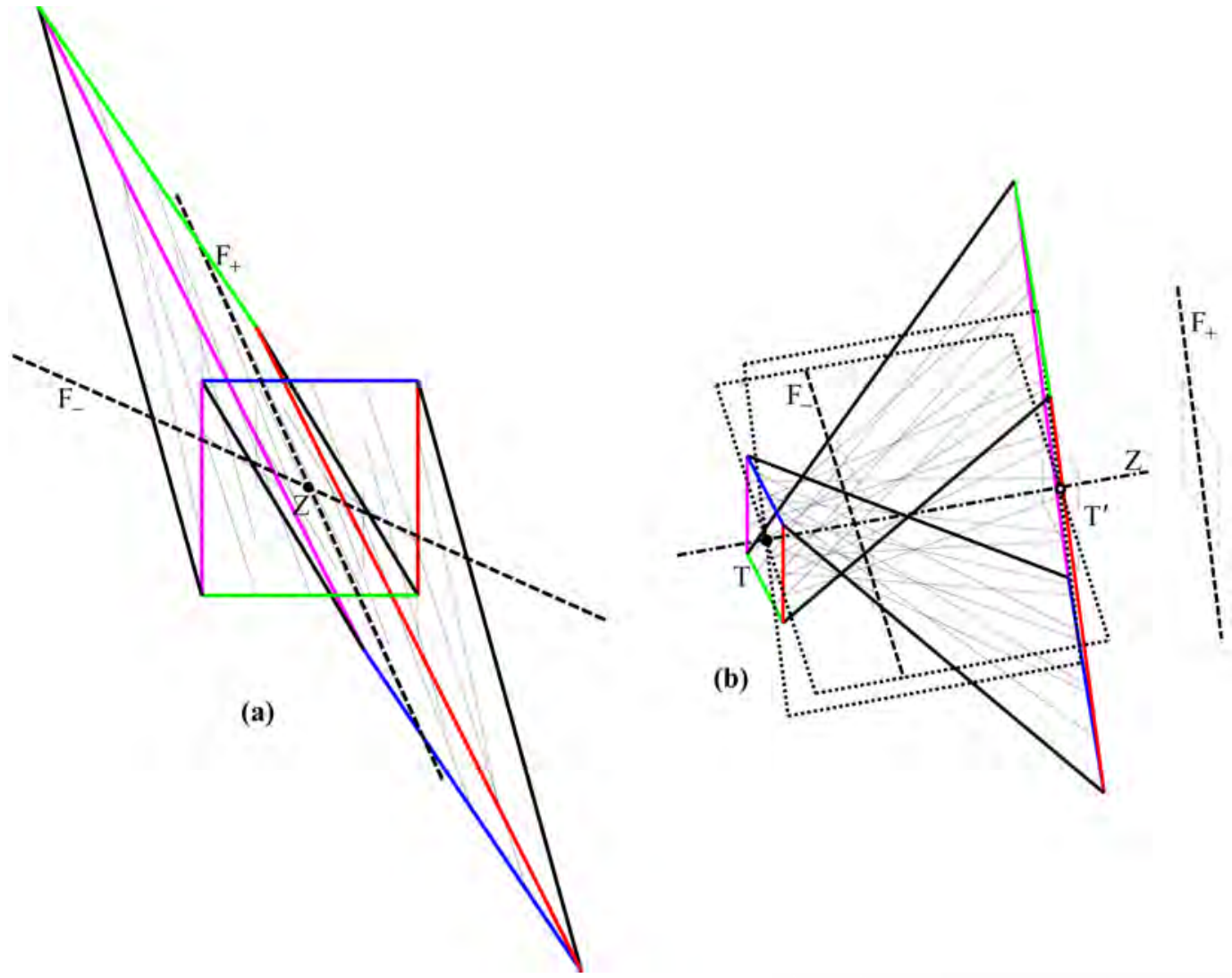

Figure 19 A pencil with divergency $\quad \mathbf{M}=-\mathbf{I}+\mathbf{J}+3 \mathbf{L} / 4 \mathrm{D}$ at $\mathrm{T}$. The second focal plane and focal line are show downstream from the exit plane at a reduced distance of about $2.95 \mathrm{~m}$. (b) azimuth $-17^{\circ}$, elevation $36^{\circ}$. 


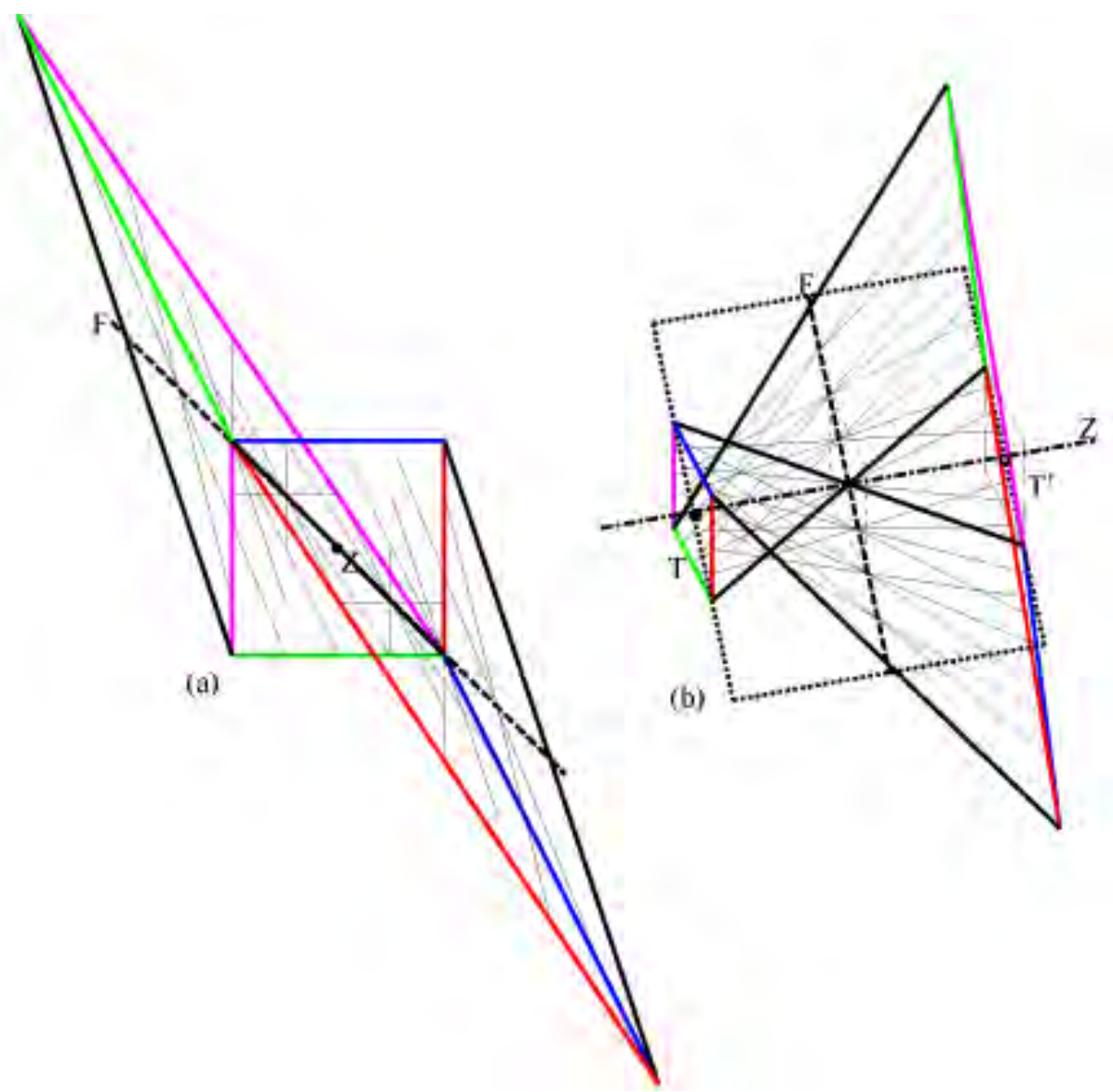

Figure 20 A pencil with divergency $\mathbf{M}=-\mathbf{I}+\mathbf{J}+\mathbf{L D}$ at T. The two principal planes of Figures 16 to 19 have merged to become one at . There is a unique focal line at the same angle. (b) azimuth $-17^{\circ}$, elevation $36^{\circ}$.
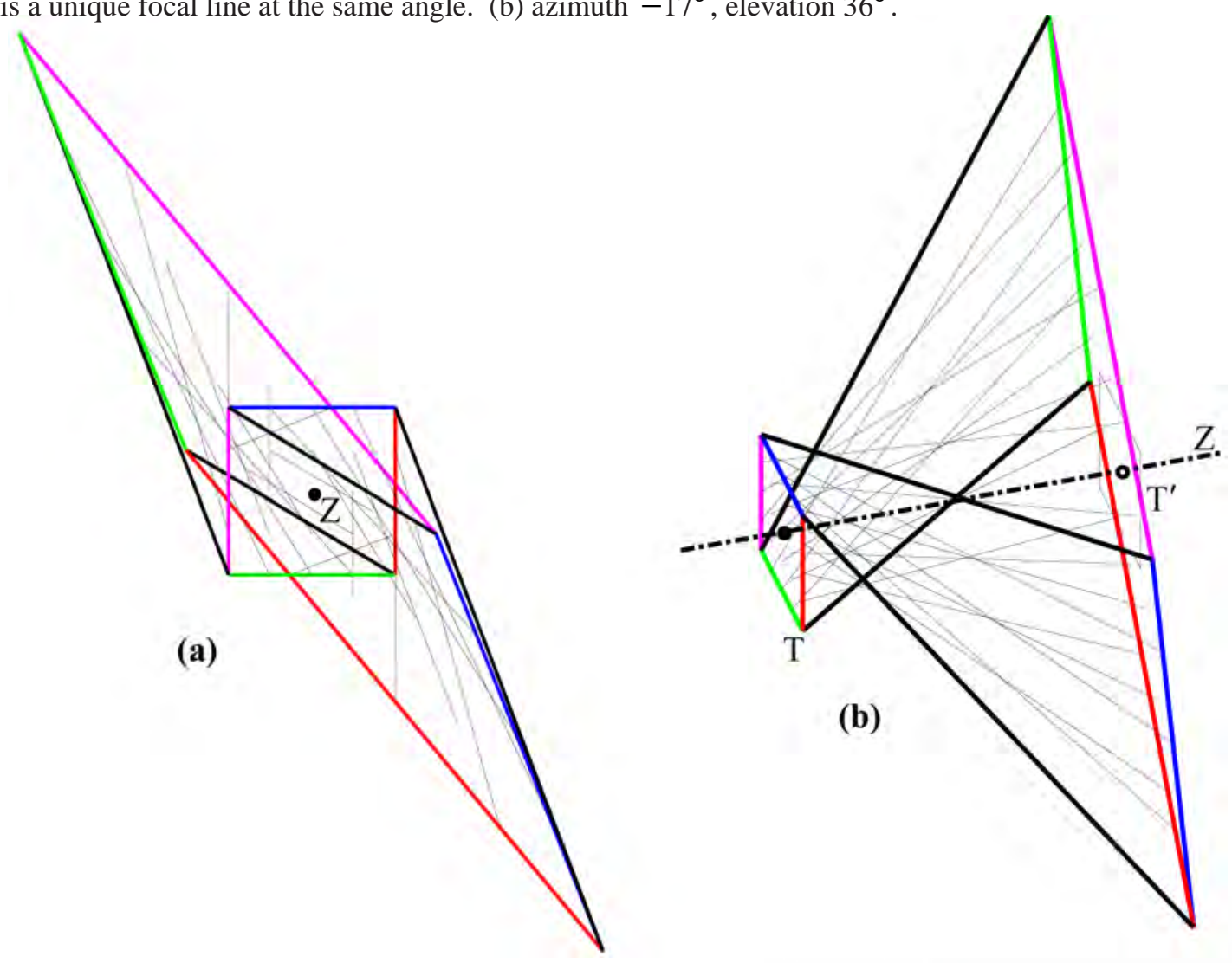

Figure 21 A pencil with divergency $\mathbf{M}=-\mathbf{I}+\mathbf{J}+5 \mathbf{L} / 4 \mathrm{D}$ at $\mathrm{T}$. There are no principal meridional planes or foci. Increasing the antistigmatic component of the divergency still further relative to that in Figure 20 has removed the line focus. (b) azimuth $-17^{\circ}$, elevation $36^{\circ}$. 


\section{Concluding remarks}

The mathematical results of the accompanying paper ${ }^{2}$ have been used to construct illustrations here of the types of pencils and associated principal meridional planes and focal planes possible in generalized optical systems. Some are pencils of the familiar type: Figure 1, a pencil of parallel rays; Figure 7, a stigmatic pencil with associated point focus; Figures 4, 10 and 13, astigmatic pencils with orthogonal principal meridional planes, orthogonal line foci and interval of Sturm; and Figure 16, a singular (cylindrical) pencil with a single finite line focus and an infinite line focus. The rest, however, are new; they are not possible in conventional optical systems. They represent new phenomena. They include pencils with no foci anywhere, not even at infinity (Figures 2, 3, 8, 9, 12 and 21); their divergencies have negative discriminants (listed in Table 1). They include pencils with single principal meridional planes and single line foci (Figures 11, 15 and 20); the discriminants are zero. And they include pencils with two principal meridional planes but the planes are not orthogonal, and nor are the two line foci (Figures 5, 6, 14, 17, 18 and 19); the discriminants are positive.

The pencils in Figures 2 and 3 have antisymmetric divergencies. Adding an antisymmetric component to the divergency of a pencil with a point focus (Figure 7) removes the focus (Figures 8 and 9). It also induces chirality in the pencil while invariance of the pencil under rotation about the longitudinal axis is preserved if the square arrangement of the rays is ignored. In the case of an astigmatic pencil (Figures 4, 10 and 13), with usual interval of Sturm, addition of an antisymmetric component results in symmetric scissors-like rotation of the principal meridional planes and line foci away from orthogonality (Figures 5, 6, 10, 14 and 15). Eventually the two planes merge into a single principal meridional plane which bisects the angle between the original planes and the two focal lines become a single focal line (Figures 11 and 15). The single focal line disappears leaving no focus if the antisymmetric component is increased still further (Figures 12). An increasing antisymmetric component makes the infinite focal plane of a singular (cylindrical) divergency (Figure 16) finite (Figure 17) and brings the two focal planes towards one another (Figures 18 and 19) which combine into one with a single focal line (Figure 20) which disappears leaving no focus (Figure 21).

One can imagine producing each of the pencils illustrated in this paper by placing a thin lens of divergence $\mathbf{C}$ equal to the divergency $\mathbf{M}$ immediately upstream of entrance plane T. By Equation $24^{2}$ parallel rays (divergency $\mathbf{O} \mathrm{D}$ and illustrated in Figure 1) incident onto the lens would result in a pencil with divergency $\mathbf{M}$ at T.

\section{Acknowledgements}

Support from the National Research Foundation for W F Harris is gratefully acknowledged. All figures were drawing using MATLAB.

\section{References}

1. Harris WF, van Gool RD. Thin lenses of asymmetric power. S Afr Optom 200968 52-60.

2. Harris WF. Ray pencils of general divergency. S Afr Optom 200968 97-110.

3. Fick HH. Fortschrittliche Rechnungsarten in der Augenoptik. Folge 20. Der Augenoptiker 1973 (12) 55-61.

4. Keating MP. Lens effectivity in terms of dioptric power matrices. Am J Optom Physiol Opt 198158 1154-1160.

5. Keating MP. A system matrix for astigmatic optical systems: I. Introduction and dioptric power relations. Am J Optom Physiol Opt 198158 810-819.

6. Harris WF. Wavefronts and their propagation in astigmatic optical systems. Optom Vis Sci 199673 606-612.

7. Harris WF. Step-along vergence procedures in stigmatic and astigmatic systems. Ophthal Physiol Opt 200020 487493.

8. Harris WF. Interconverting the matrix and principal meridional representations of dioptric power in general including powers with nonorthogonal and complex principal meridians. Ophthal Physiol Opt 200121 247-252.

9. Harris WF. Interpreting nonorthogonal keratometric measurements. Ophthal Physiol Opt 200121 253-254.

10. Harris WF. Nodes and nodal points and lines in eyes and other optical systems. Ophthal Physiol Opt 200929 in press. 